NASA/CR-2000-210557

ICASE Report No. 2000-43

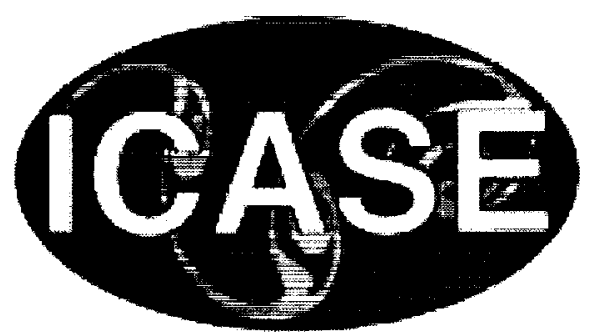

\title{
An Energy Decaying Scheme for Nonlinear Dynamics of Shells
}

Carlo L. Bottasso

Politecnico di Milano, Milano, Italy

Olivier A. Bauchau and Jou-Young Choi

Georgia Institute of Technology, Atlanta, Georgia 


\section{The NASA STI Program Office ... in Profile}

Since its founding, NASA has been dedicated to the advancement of aeronautics and space science. The NASA Scientific and Technical Information (STI) Program Office plays a key part in helping NASA maintain this important role.

The NASA STI Program Office is operated by Langley Research Center, the lead center for NASA's scientific and technical information. The NASA STI Program Office provides access to the NASA STI Database, the largest collection of aeronautical and space science STI in the world. The Program Office is also NASA's institutional mechanism for disseminating the results of its research and development activities. These results are published by NASA in the NASA STI Report Series, which includes the following report types:

- TECHNICAL PUBLICATION. Reports of completed research or a major significant phase of research that present the results of NASA programs and include extensive data or theoretical analysis. Includes compilations of significant scientific and technical data and information deemed to be of continuing reference value. NASA's counterpart of peer-reviewed formal professional papers, but having less stringent limitations on manuscript length and extent of graphic presentations.

- TECHNICAL MEMORANDUM. Scientific and technical findings that are preliminary or of specialized interest, e.g., quick release reports, working papers, and bibliographies that contain minimal annotation. Does not contain extensive analysis.

- CONTRACTOR REPORT. Scientific and technical findings by NASA-sponsored contractors and grantees.
- CONFERENCE PUBLICATIONS. Collected papers from scientific and technical conferences, symposia, seminars, or other meetings sponsored or cosponsored by NASA.

- SPECIAL PUBLICATION. Scientific, technical, or historical information from NASA programs, projects, and missions, often concerned with subjects having substantial public interest.

- TECHNICAL TRANSLATION. Englishlanguage translations of foreign scientific and technical material pertinent to NASA's mission.

Specialized services that complement the STI Program Office's diverse offerings include creating custom thesauri, building customized data bases, organizing and publishing research results ... even providing videos.

For more information about the NASA STI Program Office, see the following:

- Access the NASA STI Program Home Page at http://www.sti.nasa.gov

- Email your question via the Internet to help@sti.nasa.gov

- Fax your question to the NASA STI Help Desk at (301) 621-0134

- Telephone the NASA STI Help Desk at (301) 621-0390

- Write to: NASA STI Help Desk NASA Center for AeroSpace Information 7121 Standard Drive Hanover, MD 21076-1320 
NASA/CR-2000-210557

ICASE Report No. 2000-43

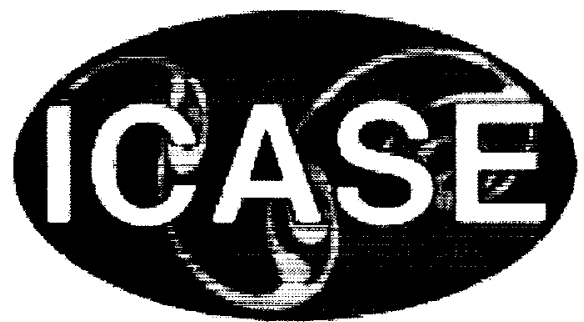

\section{An Energy Decaying Scheme for Nonlinear Dynamics of Shells}

Carlo L. Bottasso

Politecnico di Milano, Milano, Italy

Olivier A. Bauchau and Jou-Young Choi

Georgia Institute of Technology, Atlanta, Georgia

ICASE

NASA Langley Research Center

Hampton, Virginia

Operated by Universities Space Research Association

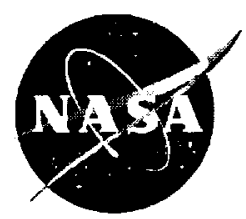

National Aeronautics and

Space Administration

Langley Research Center 
Available from the following:

NASA Center for AeroSpace Information (CASI) 7121 Standard Drive

Hanover, MD 21076-1320

(301) 621-0390
National Technical Information Service (NTIS)

5285 Port Royal Road

Springfield, VA 22161-2171

(703) $487-4650$ 


\title{
AN ENERGY DECAYING SCHEME FOR NONLINEAR DYNAMICS OF SHELLS
}

\author{
CARIO L. BOTTASSO*, OLIVIFR A. BAUCHAU ${ }^{\dagger}$, AND JOU-YOUNG CHOI ${ }^{\ddagger}$
}

\begin{abstract}
A novel integration scheme for nonlinear dynamics of geometrically exact shells is developed based on the inextensible director assumption. The new algorithm is designed so as to imply the strict decay of the system total mechanical energy at each time step, and consequently unconditional stability is achieved in the nonlinear regime. Furthermore, the scheme features tunable high frequency numerical damping and it is therefore stiffly accurate. The method is tested for a finite element spatial formulation of shells based on mixed interpolations of strain tensorial components and on a two-parameter representation of director rotations. The robustness of the scheme is illustrated with the help of numerical examples.
\end{abstract}

Key words. geometrically exact shell, time integration, energy decaying scheme

Subject classification. Applied and Numerical Mathematics

1. Introduction. The formulation of integration algorithms for nonlinear dynamirs of geometrically exact shells is the focus of this work. The partial differential equations governing this class of problems are known to present a rich mathematical structure. In particular, the resulting models are Hamiltonian systems characterized by a symplectic nature and associated with conservation laws that stem from symmetries of the Hamiltonian. The linear and angular momentum as well as the total mechanical energy are conserved for free motions of such systems.

The understanding of the geometric characteristics of the governing equations has been historically confined to the fields of analytical mechanics and pure mathematics. Surprisingly, this knowledge has been seldom used for the development of numerical methods. Indeed, the study of new integration algorithms has been traditionally preoccupied with the development of methods applicable to vast classes of problems, for example the class of differential/algebraic equations, or hyperbolic conservation laws. Consequently, classical methods rarely preserve the underlying structure of the problem being solved, and hence, such structure is lost in the numerical solution.

This approach also limits the possible theoretical analyses of the schemes, which are, more often than not, confined to linear or model cases. For instance, it is customary to characterize integration schemes for structural dynamics by studying their behavior when applied to a linear oscillator. This approach is clearly not adequate when dealing with highly nonlinear problems such as the dynamics of geometrically exact shells.

A new approach to the design of integration algorithms attempts to bridge the divide between theoretical and numerical mechanics. Under this new paradigm, numerical schemes are "backward-engineered" to preserve some important qualitative features of the governing equations. Fittingly, this approach is now called geometric integration in the mathematical community [18]. Attempts at designing "geometry-aware" algorithms for structural dynamics problems can be traced back to the work of Simo and co-workers who

\footnotetext{
- Dipartimento di Ingegneria Aerospaziale, Politecnico di Milano, Via Ta Masa 34, 20158 Milano, Italy (email address: Carlo.Bottasso@polimi.it). This research was supported by the National Aeronautics and Space Administration under NASA Contract No. NAS1-97046 while the first author was in residence at ICASF, NASA Jangley Research Center, Hampton, VA 23681-2199.

${ }^{\dagger}$ Georgia Institute of Terhnology, School of Acrospace Engineering, Atlanta, GA, USA (email address: Olivier. Bauchan@aerospace gatech.edu).

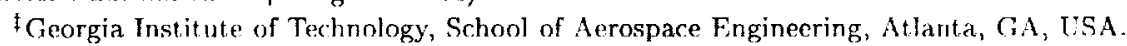


analyzed the problems of rigid body dynamics [26], nonlinear elasto-dynamics [23], geometrically exact shells [24], and geometrically exact beams [25]. In all cases, the idea was to design algorithms that ensure the discrete preservation of the total mechanical energy and of the linear and angular momenta of the system.

When integrating linear and nonlinear finite element models, the implications of the discrete equations stiffness must be carefully considered. Indeed, high frequencies are an artifact of the spatial discretization process and do not reflect the high frequencies of the original infinite dimensional problem. The need for high frequency numerical dissipation has been recognized in the past for linear problems [20]. When dealing with complex nonlinear systems, numerical dissipation becomes indispensable. Indeed, nonlinearities provide a mechanism for transferring energy from low to high frequency modes. Consequently, numerical solutions feature violent oscillations of a purely numerical origin that will eventually play havoc with the convergence characteristics of the nonlinear equation solver.

Among the various geometric characteristics of shell equations, energy preservation appears to be the most important for the development of robust time integration schemes. In fact, strict energy preservation at the discrete level leads to unconditional stability in the nonlinear regime, whereas the classical approach based on the analysis of the spectral radius leads to unconditional stability in the linear regime only. An energy preserving (EP) scheme for geometrically exact shell is developed in this paper. In addition, the scheme also preserves both linear and angular momenta of the system at the discrete level. Unfortunately, prescrvation of energy and high frequency dissipation cannot coexist, unless energy is transferred from high to low frequency modes, a transfer that has no physical basis. To solve this problem, a family of energy decaying (ED) schemes that imply a controllable energy decay within each time step is proposed in this work. In geometric terms, this means that the evolution of the system is not confined to the level set of constant energy, but is allowed to drift away from it in a monotonic and controllable manner. Since the energy remains bounded at all times, the scheme is unconditionally stable for nonlinear systems. Furthermore, it can be shown that the energy dissipation mechanism of the algorithm is the result of the removal of the higher frequencies from the computed response.

In related papers, various energy preserving and decaying geometric integrators were developed for rigid bodies and geometrically exact beams $[10,11,14,6,7]$, and nonlinear elastodynamics [9]. The concepts were extended to multibody systems featuring nonlinear holonomic constraints $[3,15,12,16,13]$; nonholonomic and unilateral constraints were treated in $[5,4]$. The integration of the present shell model in a general finite element based multibody framework is discussed in [8]. The proposed scheme is independent of the choice of spatial discretization applied to the governing partial differential equations. In the present implementation, the finite element method is used, and the mixed interpolation of tensorial components [ 1 , $2,17]$ is implemented to avoid the shear locking problem. The orientation of unit shell directors is described by a special family of two-parameter rotations.

The paper is laid out as follows. The classical equations of motion for geometrically exact shells based on inextensible unit directors are presented in section 2. Next, an EP scheme is developed in section 3. Section 4 then presents an ED algorithm with tunable high frequency dissipation that is constructed from the EP scheme. Finally, numerical examples are presented in section 5 to demonstrate the efficiency and robustness of the proposed scheme. A discussion section concludes the paper.

\section{Formulation of the Equations of Motion.}

2.1. Kinematics of the Shell Problem. Consider a shell of thickness $h$ and reference surface area $\Omega$, as depicted in fig. 2.1. An inertial frame of reference $\mathcal{S}$ consisting of three mutually orthogonal unit vectors $\mathbf{i}_{1}, \mathbf{i}_{2}, \mathbf{i}_{3}$ is used. Let $\underline{r}_{0}$ be the position vector of an arbitrary point on the reference surface of the shell, and 


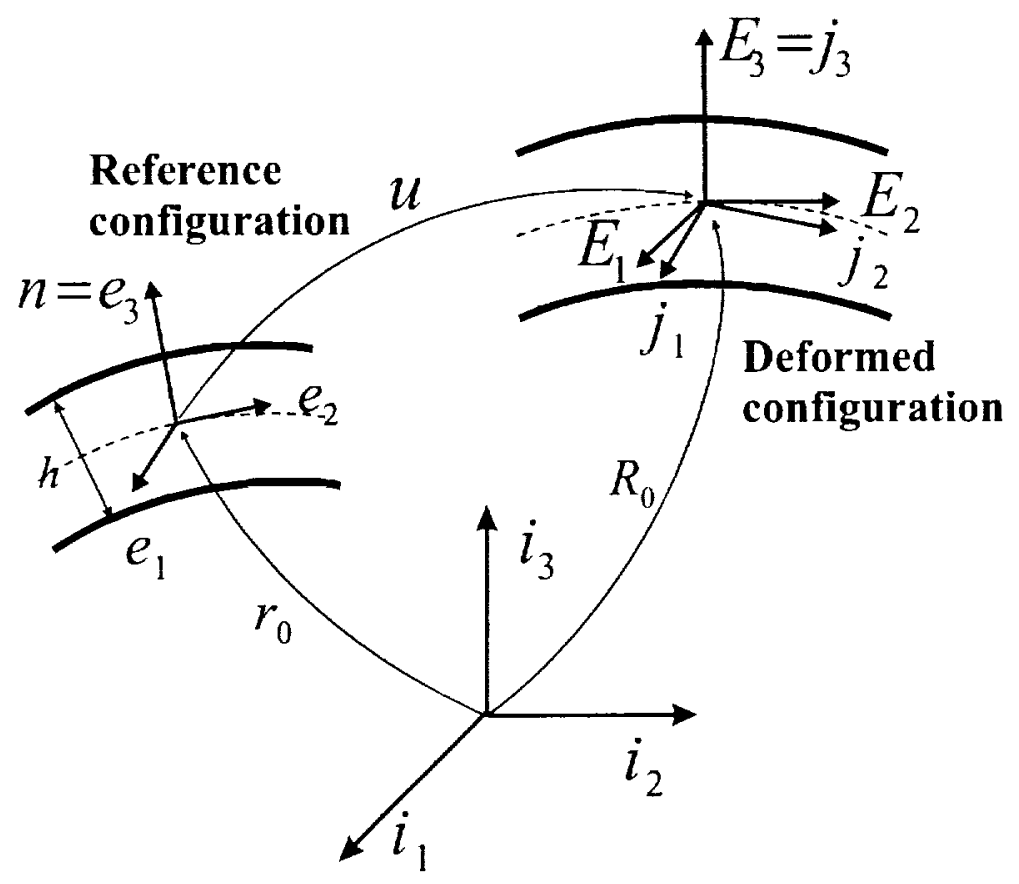

Fic: 2.1. Configuration of the shell in the reference and deformed configurations.

let $\zeta$ be the material coordinate along $\underline{n}$, the normal to the reference surface. The position vector $\underline{r}$ of an arbitrary point on the shell in its reference configuration is then

$$
\underline{r}\left(\xi^{1}, \xi^{2}, \zeta\right)=\underline{r}_{0}\left(\xi^{1}, \xi^{2}\right)+\zeta \underline{n}\left(\xi^{1}, \xi^{2}\right)
$$

where $\xi^{\prime}$ and $\xi^{2}$ are the material coordinates used to represent the shell reference surface. The coordinates $\xi^{1}, \xi^{2}$ and $\zeta$ form a set of curvilinear coordinates that are a natural choice to represent the shell geometry. The coordinates $\xi^{1}$ and $\xi^{2}$ are assumed to be lines of curvatures of the shell reference surface. The base vectors are then

$$
g=\left[\underline{g}_{1}, \underline{g}_{2}, \underline{g}_{3}\right]=\left[\underline{r}_{1}, \underline{r}_{2}, \frac{\partial \underline{r}}{\partial \zeta}\right]=\left[\left(1-\frac{\zeta}{R_{1}}\right) \underline{a}_{1},\left(1-\frac{\zeta}{R_{2}}\right) \underline{a}_{2}, \underline{n}\right],
$$

where $R_{1}$ and $R_{2}$ are the principal radii of curvature, $\underline{a}_{\alpha}=\underline{r}_{0, \alpha}$, and the notation $(\cdot), \alpha$ is used to denote a derivative with respect to $\xi^{\alpha}$. It is convenient to introduce a set of three mutually orthogonal unit vectors at the shell reference surface (i.e. at $\zeta=0$ )

$$
\underline{e}_{1}=\frac{\underline{a}_{1}}{\sqrt{a_{11}}} ; \quad \underline{e}_{2}=\frac{\underline{a}_{2}}{\sqrt{a_{22}}} ; \quad \underline{e}_{3}=\underline{n},
$$

where $a_{\alpha \alpha}=\underline{a}_{\alpha} \cdot \underline{a}_{\alpha}$.

Two fundamental assumptions will be made concerning the deformation of the shell, i.e. the material line initially normal to the reference surface of the shell remains a straight line and suffers no extension. This is the classical inextensible director model. With these assumptions, the position vector of a material point of the shell writes

$$
\underline{R}\left(\xi^{1}, \xi^{2}, \zeta\right)=\underline{r}_{0}\left(\xi^{1}, \xi^{2}\right)+\underline{u}\left(\xi^{1}, \xi^{2}\right)+\zeta \underline{E}_{3}\left(\xi^{1}, \xi^{2}\right)
$$


where $\underline{u}\left(\xi^{1}, \xi^{2}\right)$ is the reference surface displacement vector. In the deformed configuration, the base vectors at the shell reference surface are

$$
G=\left[\underline{G}_{1}, \underline{G}_{2}, \underline{G}_{3}\right]=\left[\underline{R}_{1}, \underline{R}_{, 2}, \frac{\partial \underline{R}}{\partial \zeta}\right] .
$$

Introducing the position vector, eq. (2.4), then yields

$$
\hat{G}=\left[\frac{\underline{G}_{1}}{\sqrt{a_{11}}}, \frac{\underline{G}_{2}}{\sqrt{a_{22}}}, \underline{G}_{3}\right]=E+\zeta H
$$

where

$$
E=\left[\underline{E}_{1}, \underline{E}_{2}, \underline{E}_{3}\right]=\left[\underline{e}_{1}+\frac{\underline{u}_{, 1}}{\sqrt{a_{11}}}, \underline{e}_{2}+\frac{\underline{u}_{, 2}}{\sqrt{a_{22}}}, \underline{E}_{3}\right] ; \quad H=\left[\frac{\underline{E}_{3,1}}{\sqrt{a_{11}}}, \frac{\underline{E}_{3,2}}{\sqrt{a_{22}}}, 0\right] .
$$

Note that $\underline{E}_{3}\left(\xi^{1}, \xi^{2}\right)$ is a unit vector, whereas $\underline{E}_{1}$ and $\underline{E}_{2}$ are not unit vectors, nor are they orthogonal to $\underline{E}_{3}$, as axial and transverse shearing strains develop during deformation.

2.2. Equations of Motion. The Green-Lagrange strain tensor $e$ is defined as

$$
e=\frac{1}{2}\left(G^{T} G-g^{T} g\right) \text {. }
$$

The strain tensor $e$ is defined in the curvilinear coordinate system defined by coordinates $\xi^{1}, \xi^{2}$ and $\zeta$. However, it is more convenient to work with the strain tensor $e$ defined in the locally rectangular system defined by triad $\underline{e}_{1}, \underline{e}_{2}, \underline{e}_{3}$, see eqs. (2.3). For shallow shells (i.e. $\zeta / R_{1} \ll 1$ and $\zeta / R_{2} \ll 1$ ) undergoing large displacements and rotations but small strains (all strain components are assumed to be small compared to unity), the strain-displacement relationships can be written as

$$
e=\frac{1}{2}\left[E^{T} E-I+\zeta\left(E^{T} H+H^{T} E+\kappa\right)\right]
$$

where

$$
\kappa=\left[\begin{array}{ccc}
1 / R_{1} & 0 & 0 \\
0 & 1 / R_{2} & 0 \\
0 & 0 & 0
\end{array}\right]
$$

It is clear that the strains can be expressed in terms of five parameters: the three components of the displacement field $\underline{u}$ (through $\underline{E}_{1}$ and $\underline{E}_{2}$ ) and the two parameters defining the orientation of the unit director $\underline{E}_{3}$. Virtual changes in the strain energy of the structure are given by

$$
\delta V=\int_{\Omega} \int_{h} \delta \bar{V} \mathrm{~d} \zeta \mathrm{d} \Omega=\int_{\Omega} \int_{h} \delta e \cdot \tau \mathrm{d} \zeta \mathrm{d} \Omega,
$$

where $\delta \bar{V}$ is the virtual strain energy density, and $\tau$ the second Piola-Kirchhoff stress tensor. Introducing the strains, eq. (2.9), and taking into account the symmetry of the stress tensor then yields

$$
\delta \bar{V}=\delta E \cdot(E+\zeta H) \tau+\delta H \cdot \zeta E \tau .
$$

The existence of a strain energy density function $\bar{V}$ is postulated here, hence the constitutive laws are of the form $\tau=\partial \bar{V} / \partial e$.

The velocity vector of material point $P$ of the shell is obtained by differentiating the position vector, eq. (2.4), with respect to time, to find $\underline{v}=\underline{\dot{u}}+\zeta \underline{\dot{E}}_{3}$. The kinetic energy of the system is now

$$
K=\int_{\Omega} \int_{h} \bar{K} \mathrm{~d} \zeta \mathrm{d} \Omega=\frac{1}{2} \int_{\Omega} \int_{h} \rho \underline{v} \cdot \underline{v} \mathrm{~d} \zeta \mathrm{d} \Omega,
$$


where $\bar{K}$ is the kinetic energy density. Introducing the velocity vector then yields

$$
\bar{K}=\frac{1}{2} \rho\left(\underline{\dot{u}}+\zeta \underline{\dot{E}}_{3}\right) \cdot\left(\underline{\dot{u}}+\zeta \underline{E}_{3}\right)
$$

Hamilton's Principle can now be expressed as

$$
\begin{aligned}
\int_{t_{i}}^{t_{f}} \int_{\Omega} \int_{h}(\delta \bar{K}-\delta \bar{V}) \mathrm{d} \zeta \mathrm{d} \Omega \mathrm{d} t \\
\quad=\int_{t_{i}}^{t_{f}} \int_{\Omega} \int_{h}\left[\rho\left(\delta \underline{u}+\zeta \delta \underline{E}_{3}\right) \cdot\left(\underline{\ddot{u}}+\zeta \underline{\underline{E}}_{3}\right)+\delta E \cdot(E+\zeta H) \tau+\delta H \cdot \zeta E \tau\right] \mathrm{d} \zeta \mathrm{d} \Omega \mathrm{d} t
\end{aligned}
$$

Integrating through the thickness of the shell, we get

$$
\int_{t_{i}}^{t_{1}} \int_{\Omega}\left\{\delta \underline{u} \cdot\left[\underline{\dot{h}}-\left(\underline{N}_{1,1}+\underline{N}_{2,2}\right)\right]+\delta \underline{E} \cdot\left[\underline{\dot{g}}_{f}-\left(\underline{M}_{1,1}+\underline{M}_{2,2}\right)+\underline{N}_{3}\right]\right\} \mathrm{d} \Omega \mathrm{d} t=0
$$

In this expression, $\underline{h}=m \underline{\dot{u}}+s^{*} \underline{E}_{3}$, and $\underline{g}=s^{*} \underline{\dot{u}}+I^{*} \underline{\underline{E}}_{3}$ are the linear and angular momentum vectors of the shell, respectively; the mass coefficients are defined as $m=\int_{h} \rho \mathrm{d} \zeta, s^{*}=\int_{h} \rho \zeta \mathrm{d} \zeta, I^{*}=\int_{h} \rho \zeta^{2} \mathrm{~d} \zeta$. The in-plane forces are $\underline{N}_{\alpha}=\left(E \underline{N}_{\alpha}^{*}+H \underline{M}_{\alpha}^{*}\right) / \sqrt{a_{\alpha \alpha}}$, the out-of-plane forces $\underline{N}_{3}=E \underline{N}_{3}^{*}$, and the bending moments $\underline{M}_{\alpha}=\left(E \underline{M}_{\alpha}^{*}\right) / \sqrt{a_{\alpha \alpha}}$. The convected forces are $N^{*}=\left[\underline{N}_{1}^{*}, \underline{N}_{2}^{*}, \underline{N}_{3}^{*}\right]=\int_{h} \tau \mathrm{d} \zeta$, and the convected bending moments $M^{*}=\left[\underline{M}_{1}^{*}, \underline{M}_{2}^{*}, \underline{M}_{3}^{*}\right]=\int_{h} \tau \zeta \mathrm{d} \zeta$.

The equations of motion of shells could be derived from this principle by expressing the variations $\delta \underline{E}_{3}$ in terms of two components of virtual rotation.

3. Energy Preserving Scheme. Discrete equations of motion that imply discrete conservation laws for the total mechanical energy, linear momentum and angular momentum of the system will now be developed. Times $t_{i}$ and $t_{f}$ denote the initial and final times for a time step, respectively, and the subscripts $(\cdot)_{i}$ and $(\cdot)_{f}$ indicate quantities at $t_{i}$ and $t_{f}$, respectively. Furthermore, the subscript $(\cdot)_{m}$ is used to denote mid-point average quantities defined as

$$
(\cdot)_{m}=\frac{1}{2}\left[(\cdot)_{f}+(\cdot)_{i}\right] .
$$

The following matrix identity will be used extensively

$$
A_{f}^{T} B_{f}-A_{i}^{T} B_{i}=\left(A_{f}-A_{i}\right)^{T} B_{m}+A_{m}^{T}\left(B_{f}-B_{i}\right) .
$$

Hamilton's Principle, eq. (2.15), is now approximated in time in the the following manner

$$
\begin{aligned}
\int_{\Omega} \int_{h}\left\{\rho\left[\left(\underline{u}_{f}-\underline{u}_{i}\right)+\zeta\left(\underline{E}_{3 f}-\underline{E}_{3 i}\right)\right] \cdot\left[\frac{\underline{\underline{u}}_{f}-\underline{\underline{u}}_{i}}{\Delta t}+\zeta \frac{\dot{\underline{E}}_{3 f}-\underline{\dot{E}}_{3 i}}{\Delta t}\right]\right. \\
\left.+\left(E_{f}-E_{i}\right) \cdot\left(E_{m}+\zeta H_{m}\right) \tau_{n}+\left(H_{f}-H_{i}\right) \cdot \zeta E_{m} \tau_{a}\right\} \mathrm{d} \zeta \mathrm{d} \Omega=0 .
\end{aligned}
$$

The change in strain components from $t_{i}$ to $t_{f}$ is evaluated with the help of identity (3.2) to find

$$
\begin{aligned}
e_{f}-e_{i}= & \frac{1}{2}\left[\left(E_{f}-E_{i}\right)^{T}\left(E_{m}+\zeta H_{m}\right)+\left(E_{m}+\zeta H_{m}\right)^{T}\left(E_{f}-E_{i}\right)\right. \\
& \left.+\left(H_{f}-H_{i}\right)^{T} \zeta E_{m}+\zeta E_{m}^{T}\left(H_{f}-H_{i}\right)\right] .
\end{aligned}
$$


Over one time step, the strain components can be approximated as $e(\eta)=e_{m}+\eta\left(e_{f}-e_{i}\right) / 2$, where $\eta=$ $2\left(t-t_{m}\right) / \Delta t$ is the non-dimensional time. If the strain energy density function $\bar{l}$ is viewed as a function of the scalar variable $\eta$, the mean value theorem then implies the existence of a $\bar{\eta} \in[-1,1]$ such that

$$
\bar{V}_{f}=\bar{V}_{i}+\left.\frac{\partial \bar{V}}{\partial e}\right|_{\bar{\eta}} \frac{d e}{d \eta} 2=\bar{V}_{i}+\tau_{a} \cdot\left(e_{f}-e_{i}\right)
$$

This relationship defines the average second Piola-Kirchhoff stress tensor, $\tau_{n}=\partial \bar{V} /\left.\partial e\right|_{\bar{n}}$. Combining this result with eq. (3.4) then leads to

$$
\left(E_{f}-E_{i}\right) \cdot\left(E_{m}+\zeta H_{m}\right) \tau_{a}+\left(H_{f}-H_{i}\right) \cdot \zeta E_{m} \tau_{n}=\left(\rho_{f}-e_{i}\right) \cdot \tau_{a}=\bar{V}_{f}-\bar{V}_{i},
$$

where the symmetry of the stress tensor was taken into account. For linear constitutive laws of the form $\tau=C^{*} e$, where $C^{*}$ is the stiffness matrix, the average stress tensor simply becomes $\tau_{a}=C^{*} e_{m}$.

The following configuration updates are now defined

$$
\frac{\underline{u}_{f}-\underline{u}_{i}}{\Delta t}=\underline{\dot{u}}_{m} ; \quad \frac{\underline{E}_{3 f}-\underline{E}_{3 i}}{\Delta t}=\underline{\dot{E}}_{3 m}
$$

Introducing eqs. (3.6) and (3.7) into the approximate expression for Hamilton's Principle, eq. (3.3), then leads to

$$
\begin{aligned}
\int_{\Omega} \int_{h}\left\{\frac{\rho}{2}\left(\underline{u}_{m}+\zeta \underline{\dot{E}}_{3 m}\right) \cdot\left[\left(\underline{i}_{f}-\underline{\dot{u}}_{i}\right)+\zeta\left(\underline{\dot{E}}_{3 f}-\underline{\dot{E}}_{3 i}\right)\right]+\left(\bar{V}_{f}-\bar{V}_{i}\right)\right\} \mathrm{d} \zeta \mathrm{d} \Omega \\
=\int_{\Omega} \int_{h}\left\{\frac{\rho}{2}\left(\underline{\dot{u}}_{f}+\zeta \underline{\dot{E}}_{3 f}\right) \cdot\left(\underline{\dot{u}}_{f}+\zeta \underline{\dot{E}}_{3 f}\right)-\frac{\rho}{2}\left(\underline{\dot{u}}_{i}+\zeta \underline{\dot{E}}_{3 i}\right) \cdot\left(\underline{\dot{u}}_{i}+\zeta \underline{E}_{3 i}\right)+\left(\bar{V}_{f}-\bar{V}_{i}\right)\right\} \mathrm{d} \zeta \mathrm{d} \Omega \\
\quad=\int_{\Omega} \int_{h}\left[\left(\bar{K}_{f}-\bar{K}_{i}\right)+\left(\bar{V}_{f}-\bar{V}_{i}\right)\right] \mathrm{d} \zeta \mathrm{d} \Omega=0 .
\end{aligned}
$$

This result clearly implies the conservation of the total mechanical energy of the system within a step. In summary, the approximate form of Hamilton's Principle given by eq. (3.3) leads to a discrete energy conservation statement, eq. (3.8), when the configuration updates are chosen according to eqs. (3.7), and the average stress according to eq. (3.5).

Integrating through the thickness of the shell leads to

$$
\begin{aligned}
\int_{\Omega}\left\{( \underline { u } _ { f } - \underline { u } _ { i } ) \cdot \left[\frac{\underline{h}_{f}-\underline{h}_{i}}{\Delta t}-\left(\underline{N}_{1 m, 1}\right.\right.\right. & \left.\left.+\underline{N}_{2 m, 2}\right)\right] \\
& \left.+\left(\underline{E}_{3 f}-\underline{E}_{3 i}\right) \cdot\left[\frac{\underline{g}_{f}-\underline{g}_{i}}{\Delta t}-\left(\underline{M}_{1 m, 1}+\underline{M}_{2 m, 2}\right)+\underline{N}_{3 m}\right]\right\} \mathrm{d} \Omega=0 .
\end{aligned}
$$

In this expression, the in-plane forces are $\underline{N}_{\alpha m}=\left(E_{m} \underline{N}_{\alpha a}^{*}+H_{m} \underline{M}_{\alpha a}^{*}\right) / \sqrt{a_{\alpha \alpha}}$, the out-of-plane forces $\underline{N}_{3 m}=$ $E_{m} \underline{N}_{3 a}^{*}$, and the bending moments $\underline{M}_{\alpha m}=\left(E_{m} \underline{M}_{\alpha a}^{*}\right) / \sqrt{a_{\alpha r r}}$. The discrete governing equations of motion for shells are then

$$
\begin{gathered}
\frac{\underline{h}_{f}-\underline{h}_{i}}{\Delta t}-\left(\underline{N}_{1 m, 1}+\underline{N}_{2 m, 2}\right)=\underline{p}_{m} ; \\
Q_{m}^{T} \frac{\underline{g}_{f}-\underline{g}_{i}}{\Delta t}-Q_{m}^{T}\left(\underline{M}_{1 m, 1}+\underline{M}_{2 m, 2}-\underline{N}_{3 m}\right)=\underline{q}_{m}^{*},
\end{gathered}
$$

where $\underline{p}$ are the externally applied loads, and $\underline{q}^{*}$ the externally applied moments measured in the local system. The finite change in director orientation $\underline{E}_{3 f}-\underline{E}_{31}$ was expressed in terms of the two parameter incremental rotation vector, see $B$. 
Invariance of the system Hamiltonian under spatial translations and rotations implies the conservation of the linear and angular momenta. Althongh discrete preservation of momenta is less crucial than discrete preservation of energy, it is interesting to note that eqs. (3.10) and (3.11) also imply the discrete preservation of this invariant. At first, eqs. (3.10) are projected onto the test functions $\underline{\tilde{\pi}}\left(\underline{r}_{0}+\underline{u}_{m}\right)$ and eqs. (3.11) onto the test functions $\underline{\widetilde{\pi}} \underline{E}_{3 m}$, where $\underline{\pi}$ is an arbitrary vector. Next, integration over the shell reference surface yields

$$
\begin{aligned}
\int_{\Omega}\left\{\tilde { \widetilde { \pi } } ( \underline { r } _ { 0 } + \underline { u } _ { m } ) \cdot \left[\frac{\underline{h}_{f}-\underline{h}_{i}}{\Delta t}-\left(\underline{N}_{1 m, 1}\right.\right.\right. & \left.\left.+\underline{N}_{2 m, 2}\right)\right] \\
& \left.+\underline{\widetilde{\pi}} \underline{E}_{3 m} \cdot\left[\frac{\underline{g} f-\underline{g}_{i}}{\Delta t}-\left(\underline{M}_{1 m, 1}+\underline{M}_{2 m, 2}\right)+\underline{N}_{3 m}\right]\right\} \mathrm{d} \Omega=0 .
\end{aligned}
$$

Straightforward algebraic manipulations then lead to

$$
\begin{aligned}
\frac{\underline{\pi}}{\Delta t} \cdot \int_{\Omega}\left[\left(\underline{\underline{r}}_{0}+\underline{\tilde{u}}_{f}\right) \underline{h}_{f}-\left(\underline{\widetilde{r}}_{0}+\underline{\tilde{u}}_{i}\right) \underline{h}_{i}+\underline{\tilde{h}}_{m}\left(\underline{\tilde{u}}_{f}-\underline{\tilde{u}}_{i}\right)\right. & \\
& \left.+\underline{\widetilde{E}}_{f} \underline{g}_{f}-\underline{\widetilde{E}}_{i} \underline{g}_{i}+\underline{\widetilde{g}}_{m}\left(\underline{\widetilde{E}}_{f}-\underline{\widetilde{E}}_{i}\right)\right] \mathrm{d} \Omega=0
\end{aligned}
$$

where the following result was used

$$
\underline{\widetilde{E}}_{1 m} \underline{N}_{1 m}+\underline{\widetilde{E}}_{2 m} \underline{N}_{2 m}+\underline{\widetilde{E}}_{3,1 m} \underline{M}_{1 m}+\underline{\widetilde{E}}_{3,2 m} \underline{M}_{2 m}+\underline{\widetilde{E}}_{3 m} \underline{N}_{3 m}=0 .
$$

Inserting the configuration updates, eqs. (3.7), into eq. (3.13) then yiclds

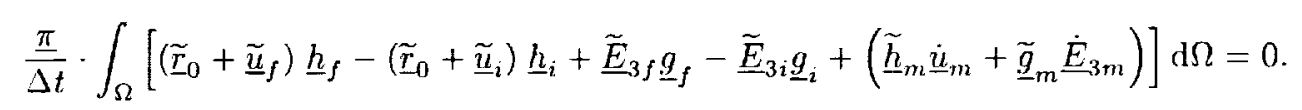

It is easily verified that $\underline{\tilde{h}}_{m} \underline{\dot{u}}_{m}+\underline{\tilde{g}}_{m} \underline{\dot{E}}_{3 m}=0$. Hence, since $\underline{\pi}$ is arbitrary, eq. (3.15) implies the discrete conservation of the total angular momentum, $\int_{\Omega}\left[\left(\underline{\tilde{r}}_{0}+\underline{\tilde{u}}\right) \underline{h}+\underline{\widetilde{E}} \underline{g}\right] \mathrm{d} \Omega$. Finally, projecting eqs. (3.10) onto the test functions $\underline{\pi}$ and eqs. (3.11) onto the null test functions gives the discrete conservation of the total linear momentum $\int_{\Omega} \underline{h} \mathrm{~d} \Omega$.

It is important to note that any spatial discretization of the discrete equations of motion will inherit the discrete energy and momentum conservation statements just proved, when the configuration updates are chosen according to eqs. (3.7), and the average stress according to eq. (3.5).

4. Energy Decaying Scheme. As discussed in the introduction, energy preservation, per se, is not sufficient to yield robust time integration schemes. High frequency numerical dissipation must be added as an inherent feature of the scheme. Such a scheme will now be constructed for the shell equations of motion using the EP scheme as a basic building block.

First, an additional state is introduced at time $t_{j}=\lim _{c \rightarrow 0}\left(t_{i}+\epsilon\right)$, and the subscript $(\cdot)_{j}$ is used to denote quantities at this time. The following averages are now defined

$$
(\cdot)_{g}=\frac{1}{2}\left[(\cdot)_{f}+(\cdot)_{j}\right] ; \quad(\cdot)_{h}=\frac{1}{2}\left[(\cdot)_{j}+(\cdot)_{i}\right] .
$$

The ED scheme proceeds from the initial to the final time by means of two coupled steps: one step from $t_{i}$ to $t_{f}$, the other from $t_{i}$ to $t_{j}$. The time-discrete equations of dynamic equilibrium are

$$
\begin{gathered}
\frac{\underline{h}_{f}-\underline{h}_{i}}{\Delta t}-\left(\underline{N}_{1, g, 1}+\underline{N}_{2 g, 2}\right)=\underline{p}_{g} ; \\
Q_{m}^{T} \frac{\underline{g}_{f}-\underline{g}_{i}}{\Delta t}-Q_{g}^{T}\left(\underline{M}_{1 g, 1}+\underline{M}_{2 g, 2}-\underline{N}_{3 g}\right)=\underline{q}_{q}^{*}
\end{gathered}
$$




$$
\begin{gathered}
\frac{\underline{h}_{j}-\underline{h}_{i}}{\Delta t}+\frac{1}{3}\left[\left(\underline{N}_{1 g, 1}+\underline{N}_{2 g, 2}\right)-\left(\underline{N}_{1 p, 1}+\underline{N}_{2 p, 2}\right)\right]=\underline{p}_{h} \\
Q_{h}^{T} \frac{\underline{g}_{j}-\underline{g}_{i}}{\Delta t}+\frac{1}{3}\left[Q_{g}^{T}\left(\underline{M}_{1 g, 1}+\underline{M}_{2 g, 2}-\underline{N}_{3 g}\right)-Q_{h}^{T}\left(\underline{M}_{1 p, 1}+\underline{M}_{2 p, 2}-\underline{N}_{3 p}\right)\right]=\underline{q}_{h}^{*} .
\end{gathered}
$$

The configuration update relationships are given as

$$
\begin{array}{ll}
\underline{u}_{f}=\underline{u}_{i}+\Delta t\left(\underline{\dot{u}}_{f}+\underline{\dot{u}}_{j}\right) / 2, & \underline{u}_{j}=\underline{u}_{i}-\Delta t\left[\underline{\dot{u}}_{f}-\underline{\dot{u}}_{i}-\alpha\left(\underline{\dot{u}}_{j}-\underline{\dot{u}}_{i}\right)\right] / 6 \\
\underline{E}_{3 f}=\underline{E}_{3 i}+\Delta t\left(\underline{\dot{E}}_{3 f}+\underline{\dot{E}}_{3 j}\right) / 2, & \underline{E}_{3 j}=\underline{E}_{3 i}-\Delta t\left[\underline{\dot{E}}_{3 f}-\underline{E}_{3 i}-\alpha\left(\underline{\dot{E}}_{3 j}-\underline{E}_{3 i}\right)\right] / 6,
\end{array}
$$

where $\alpha$ is a tuning parameter that controls the amount of numerical dissipation provided by the scheme, while the forces $\underline{N}_{\alpha p}$ and moments $\underline{M}_{\alpha p}$ are given by

$$
\underline{N}_{\alpha p}=\underline{N}_{\alpha h}+\alpha\left(\underline{N}_{\alpha j}-\underline{N}_{\alpha i}\right) / 2 ; \quad \underline{M}_{\alpha p}=\underline{M}_{\alpha h}+\alpha\left(\underline{M}_{\alpha j}-\underline{M}_{\alpha i}\right) / 2 .
$$

Using developments similar to those exposed for the EP scheme, it can be easily shown that the proposed discrete equations imply

$$
\left(K_{f}+V_{f}\right)-\left(K_{i}+V_{i}\right)+\alpha c^{2}=0
$$

$c^{2}$ is a positive quantity given by

$$
\begin{aligned}
c^{2} & =\int_{\Omega} \frac{1}{2}\left[m\|\underline{\dot{u}}\| \cdot\|\underline{\dot{u}}\|+2 s^{*}\|\underline{\dot{u}}\| \cdot\left\|\underline{\dot{E}}_{3}\right\|+I^{*}\left\|\underline{\dot{E}}_{3}\right\| \cdot\left\|\underline{E}_{3}\right\|\right] \mathrm{d} \Omega \\
& +\int_{\Omega} \frac{1}{2}\|\underline{e}\| C^{*}\|\underline{e}\| \mathrm{d} \Omega \geq 0,
\end{aligned}
$$

where $\|\cdot\|=(\cdot)_{j}-(\cdot)_{i}$ is the jump between $t_{i}$ and $t_{j}$. This result implies the decay of the total mechanical energy over one step of the algorithm, $\left(K_{f}+V_{f}\right) \leq\left(K_{i}+V_{i}\right)$. The parameter $\alpha$ clearly controls the amount of energy that is dissipated within the step. Two such parameters could be used, controlling the amount of dissipated kinetic and strain energies, respectively, but this level of complexity does not seem to be necessary. The property of preservation of momentum observed in the EP case is lost in the ED algorithm.

If the above ED scheme is applied to a single degree of freedom linear oscillator, the asymptotic value of the spectral radius of the amplification matrix, $\rho_{\infty}$, is found to be $\rho_{\infty}=(1-\alpha) /(1+\alpha)$. For $\alpha=1$, $\rho_{\infty}=0$, and asymptotic annihilation is achieved. If $\alpha=0, \rho_{\infty}=1$, and in view of eq. (4.6), energy is exactly preserved. Hence, the ED scheme is in fact a family of schemes with a single tuning parameter, $\alpha$, that controls the amount of high frequency numerical dissipation; both asymptotic annihilation or exact energy preservation can be achieved with the same scheme by using $\alpha=1$ or 0 , respectively.

5. Numerical Examples. All the examples described in this section will be treated with the proposed ED family of schemes corresponding to values of the tuning parameter $\alpha \in[0,1]$. Although any value of $\alpha$ within this range can be used, the examples described here will contrast the two extreme choices. For $\alpha=1$ ( $\rho_{\infty}=0$ ), asymptotic annihilation is obtained, and this will be called the ED scheme. On the other hand; for $\alpha=0\left(\rho_{\infty}=1\right)$, exact energy preservation is achieved, and this will be called the EP scheme.

5.1. Clamped Half-Cylinder under Point Load. Consider the half-cylinder of radius $R=1.2 \mathrm{~m}$ and width $b=2 \mathrm{~m}$ depicted in fig. 5.1. The shell has a thickness $t=6 \mathrm{~mm}$, is build-in along edge $B C$ and free along the other. The structure is made of aluminum; Young's modulus $E=73 \mathrm{GPa}$, Poisson's 


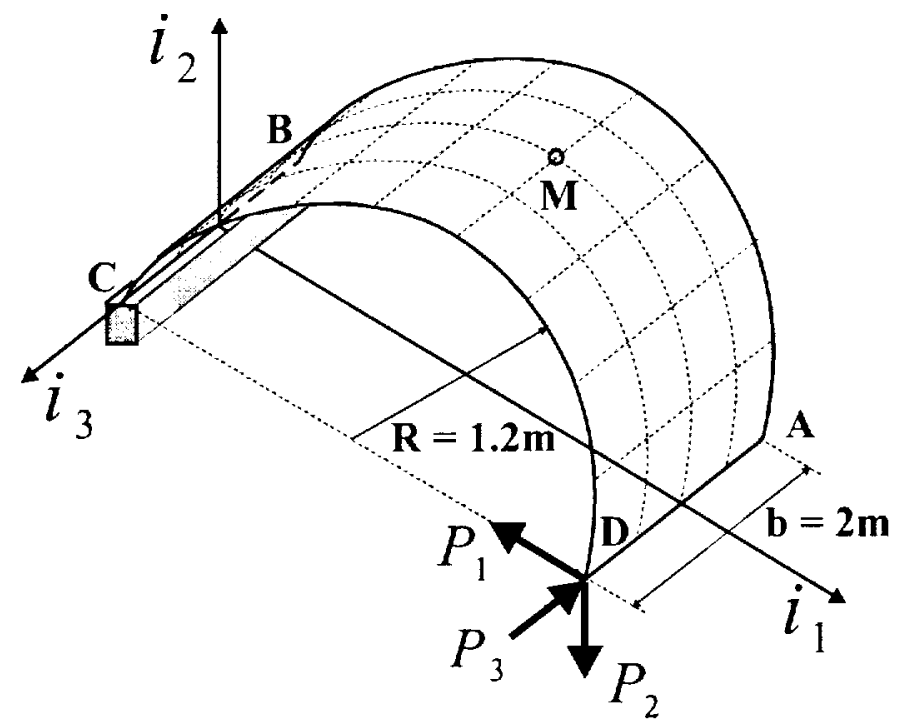

Fis: 5.1. Configuration of the clamped half-cylinder.

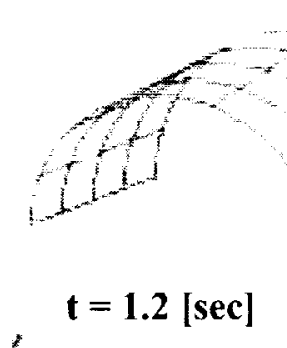

(1)

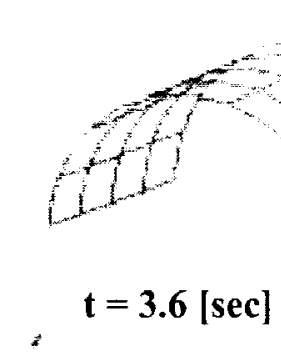

$+\ldots$

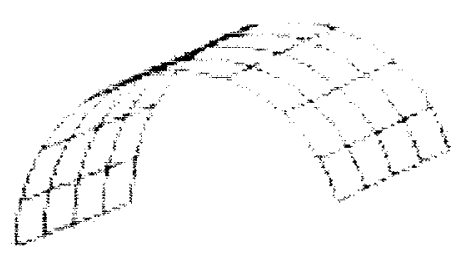

$$
\mathrm{t}=\mathbf{2 . 4}[\mathrm{sec}]
$$

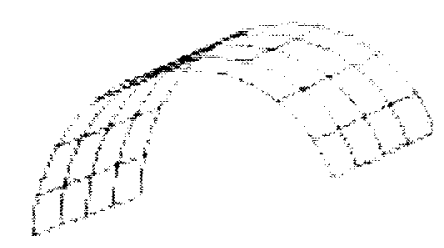

$$
\mathrm{t}=\mathbf{4 . 8}[\mathrm{sec}]
$$

FIG. 5.2. Configuration of the system at various instants in time.

ratio $\nu=0.30$ and density $\rho=2700 \mathrm{~kg} / \mathrm{m}^{3}$. At point $D$, the shell is subjected to a concentrated load $\underline{P}=-P_{0}(t)\left(\underline{i}_{1}+\underline{i}_{2}+\underline{i}_{3}\right)$. The magnitude of the load is

$$
P_{0}(t)= \begin{cases}P(1-\cos 2 \pi t / T) / 2 & t \leq T, \\ 0 & t>T,\end{cases}
$$

where $P=0.1 \mathrm{kN}$ and $T=2.0 \mathrm{~s}$. The shell was modeled by a regular $8 \times 4$ mesh of quadratic elements. All simulations were run with a time step $\Delta t=5.010^{-03} \mathrm{~s}$, for a total time of $6 \mathrm{~s}$.

Under the effect of the applied loads, the shell bends predominantly in the vertical direction, its direction 


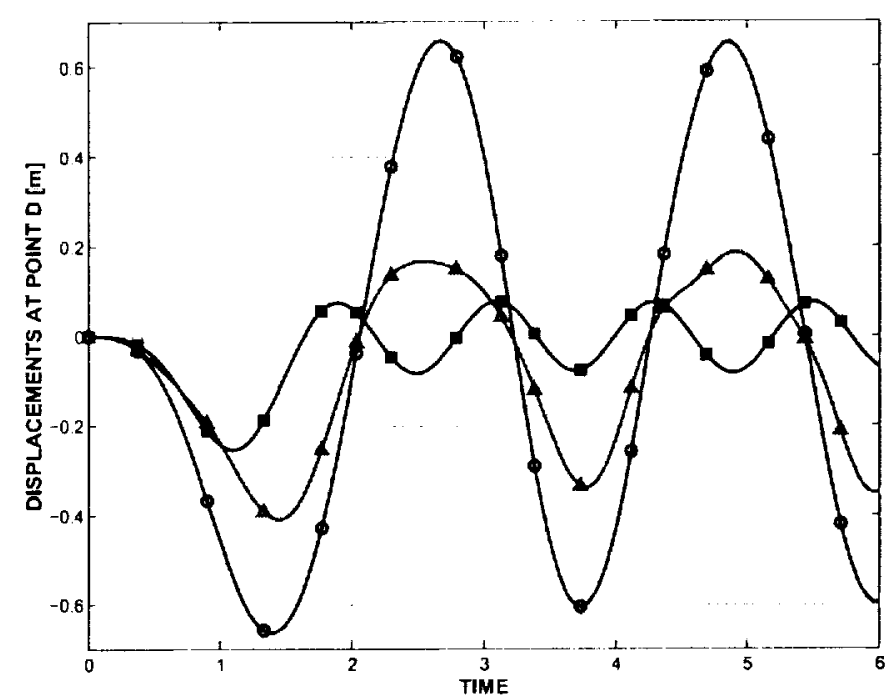

FIG. 5.3. Displacement components at point D. $U_{1}: A ; U_{2}: 0 ; U_{3}: \square$. FN scheme: solid line; EP scheme: dashed line.

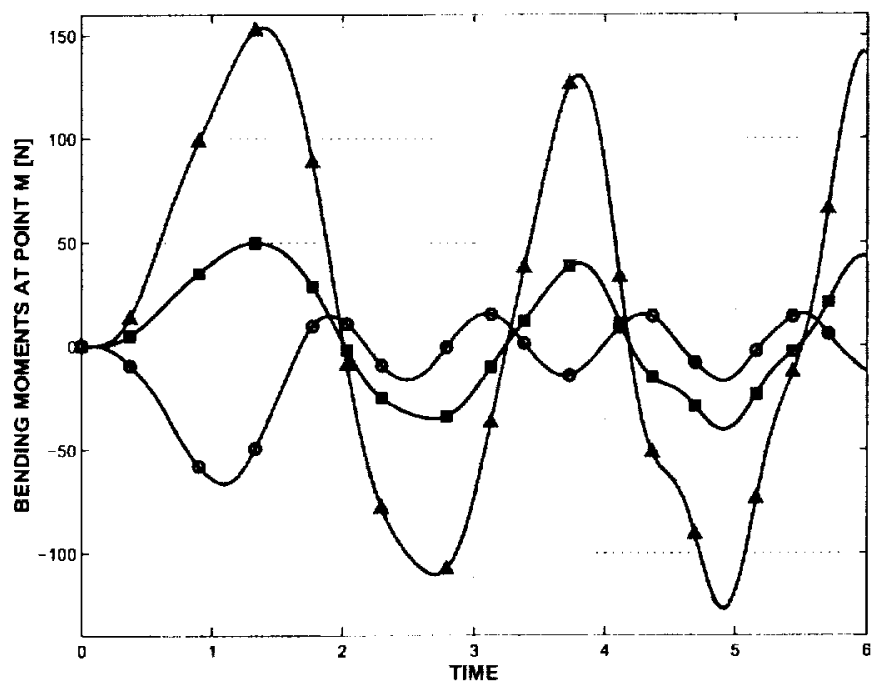

FIc. 5.4. Time history of bending moments at point $M . M_{11}: \triangle ; M_{12}: 0 ; M_{22}: \square$. ED scheme: solid line; FP scheme: dashed line.

of least bending stiffness, as illustrated in fig. 5.2 that shows the configuration of the system at various instants in time. The three components of displacements at point $D$ are shown in fig. 5.3; vertical displacements of up to $0.6 \mathrm{~m}$ are observed. The bending and twisting moments, $M_{11}, M_{22}$, and $M_{12}$, respectively, at point $M$ are shown in fig. 5.4. Note the significant transverse and twisting moments associated with the three-dimensional motion of the shell. The components of in-plane and transverse shearing forces are shown in fig. 5.5 and 5.6 , respectively.

Next, the same problem was simulated with $\rho_{\infty}=1$, i.e. with no high frequency dissipation. The corresponding results are shown in figs. 5.3 to 5.6. Displacement and moment results are found to be in excellent agreement. At the scale of the figures, they are, in fact, indistinguishable. For the period of time $2<t<6 \mathrm{~s}$, the system is not subjected to any loading, and the total mechanical energy of the system should remain constant. For the EP scheme, the energy is indeed preserved, as expected; for the ED scheme, $0.3 \%$ 

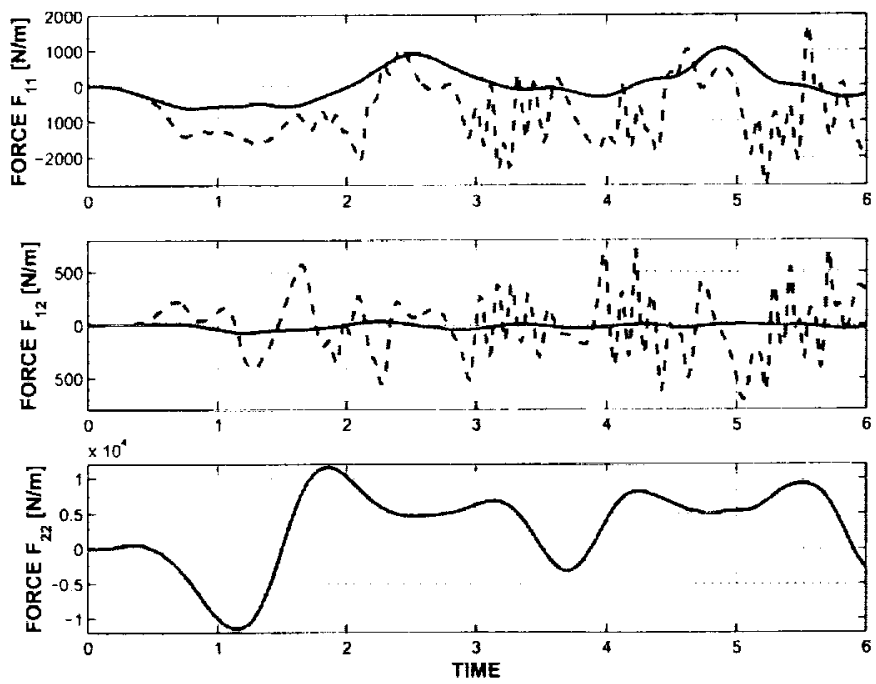

Fic. 5.5. Time history of in-plane forces at point $M . F_{11}$ : top figure; $F_{12}:$ middle figure; $F_{22}:$ bottom figure. EN scheme: solid line; EP scheme: dashed line.
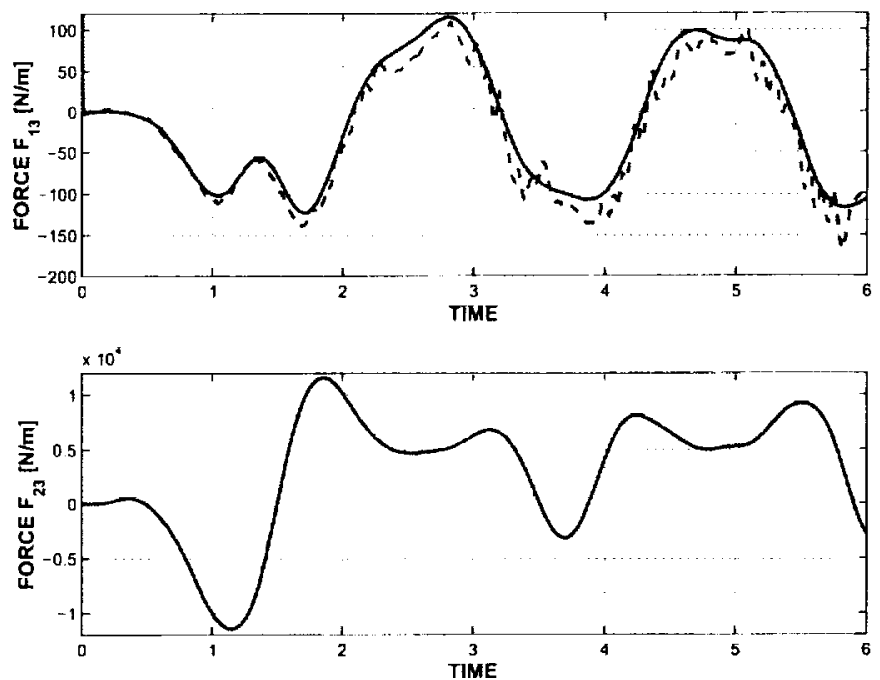

FIG. 5.6. Time history of transverse shear forces at point $M . F_{13}$ : top figure; $F_{23}$ : bottom figure. En scheme: solid line; FP scheme: dashed line.

of the energy is numerically dissipated in this period. It could be concluded that the EP and ED solutions are nearly identical, and that numerical dissipation is not necessary. However, the ED and EP scheme predictions for the in-plane and transverse shearing forces, shown in fig. 5.5 and 5.6, are markedly different. EP predictions for force components $F_{11}, F_{12}$, and $F_{13}$ show high frequency oscillations that are absent in the corresponding ED predictions. A simulation using the ED scheme with a time step $\Delta t=1.010^{-03} \mathrm{~s}$ showed that the ED predictions are converged. A simulation using the EP scheme and the same smaller time step yielded results with increased high frequency oscillations for the force predictions. It should be noted that the dynamic response of this simple system is very smooth; yet even here, high frequency numerical dissipation appears to be necessary to obtain a smooth, converged solution. 


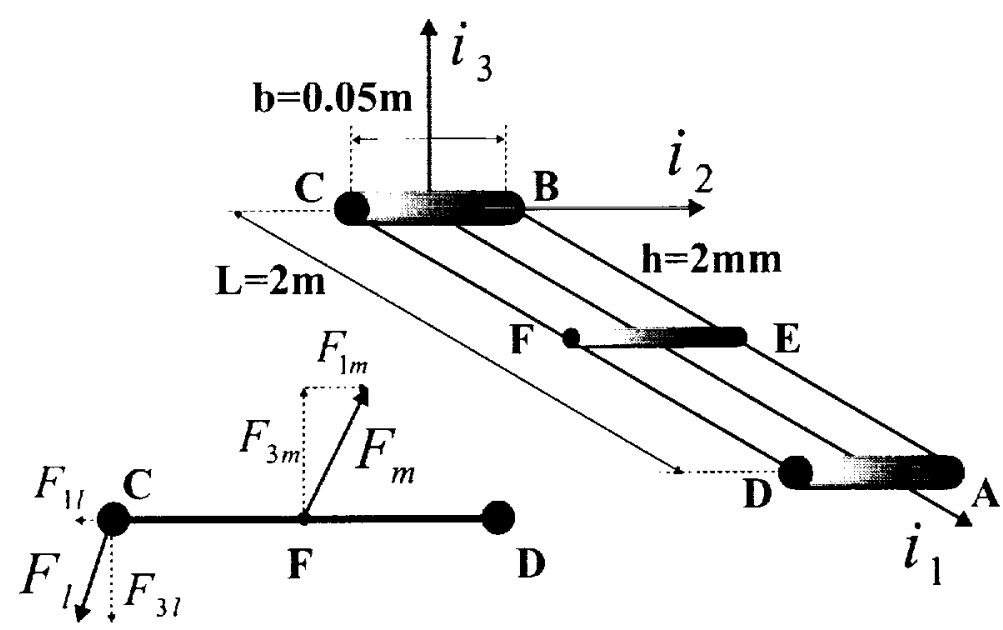

FIG. 5.7. Configuration of the plate with concentrated masses.
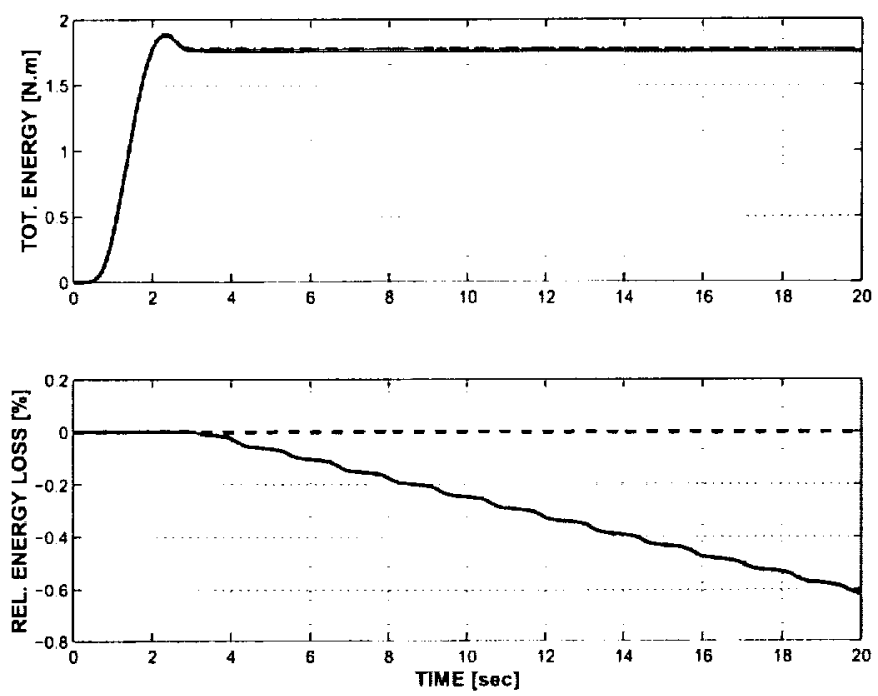

Fig. 5.8. Time history of the total mechanical energy (top figure) and relative energy loss (bottom figure). Plate model (FDS): solid line; Plate model (EPS): dashed line; Beam model (EDS): dashed-dot line.

5.2. Dynamic Response of a Plate with Edge Beams. Consider the rectangular plate of length $L=2 \mathrm{~m}$, width $b=0.05 \mathrm{~m}$ and thickness $t=2 \mathrm{~mm}$ as depicted in fig. 5.7 . Two circular beams of radius $r=2 \mathrm{~mm}$ are attached at the plate edges. A third circular beam is located at the center of the plate. All components are made of aluminum; Young's modulus $E=73 \mathrm{GPa}$, density $\rho=2700 \mathrm{~kg} / \mathrm{m}^{3}$. The total mass of the edge beams is $10 \mathrm{~kg}$ each, and that of the central beam is $1 \mathrm{~kg}$. The plate is subjected to uniformly distributed loads $F_{m}$ and $F_{f}$ along $F E$ and $C B$, respectively. The components of these loads along the $\underline{i}_{1}$ and $\underline{i}_{3}$ axes are $F_{1 m}-40 \mathrm{~N} / \mathrm{m}$ and $F_{3 m}=80 \mathrm{~N} / \mathrm{m}$, respectively, and $F_{1 \ell}=-20 \mathrm{~N} / \mathrm{m}$ and $F_{3 \ell}=-60 \mathrm{~N} / \mathrm{m}$, respectively. The common time history of each loading component is

$$
F_{i}(t)= \begin{cases}F_{i}(1-\cos 2 \pi t / T) / 2 & t \leq T \\ 0 & t>T\end{cases}
$$




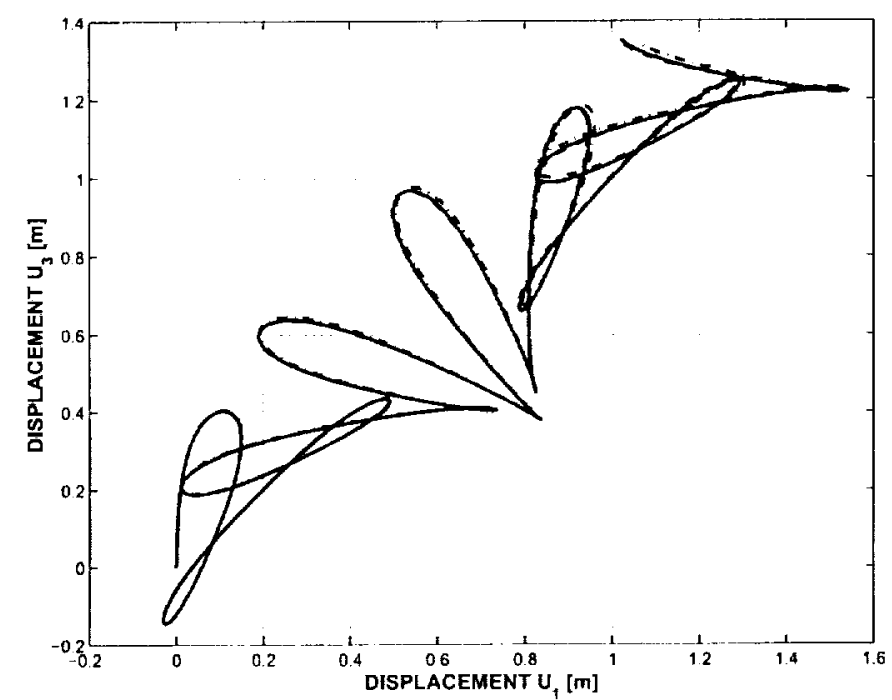

FIG. 5.9. Trajectory of the plate mid-span point. Plate model (EDS): solid line; Plate model (EPS): dashed line; Beam model (EDS): dashed-dot line.

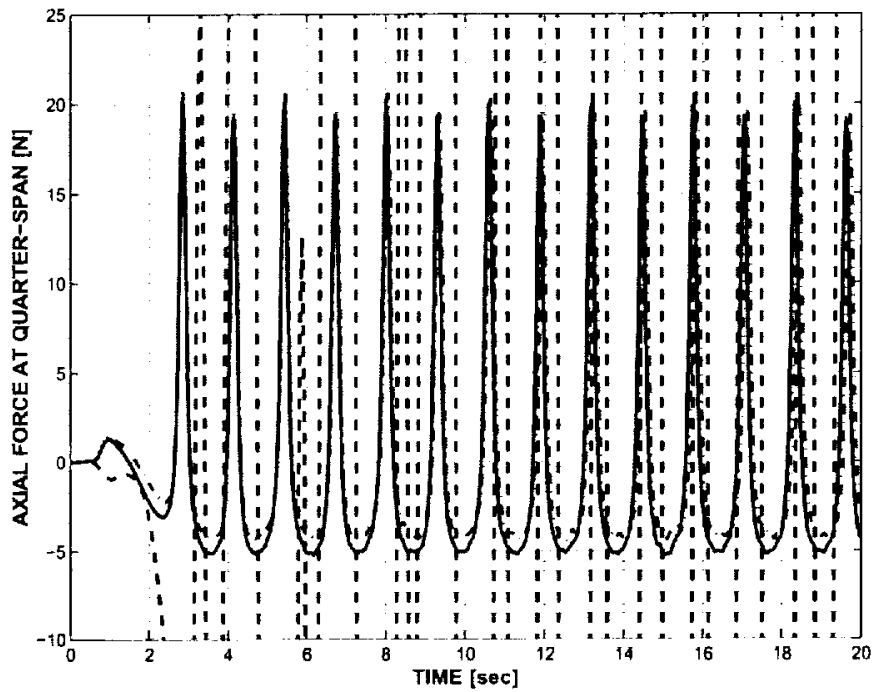

FIG. 5.10. Time history of the quarter-span axial force. Plate model (EDS): solid line; Plate model (EPS): dashed line; Beam model (EDS): dashed-dot line.

where $T=3.0 \mathrm{~s}$. The plate is discretized with 10 quadratic plate elements along its length. A constant time step $\Delta t=610^{-03} \mathrm{~s}$ was used for all simulations.

At time $t>3 \mathrm{~s}$ the applied load vanishes, and the system total mechanical energy should remain constant. The evolution of the energy of the system for three different cases is shown in fig. 5.8: the plate model using the ED scheme, the same plate model using the EP scheme, and a simplified model of the system using beam elements and the ED scheme. For times $t>3 \mathrm{~s}$, the total energy remains a constant for the EP scheme and nearly constant for the ED schemes. The relative energy loss is also presented in the figure: $0.6 \%$ of the energy was dissipated by the ED scheme in the period $t \in[3,20] \mathrm{s}$. This figure clearly demonstrates the non-increasing property of the energy evolution for the proposed ED schemes, as opposed the constant 


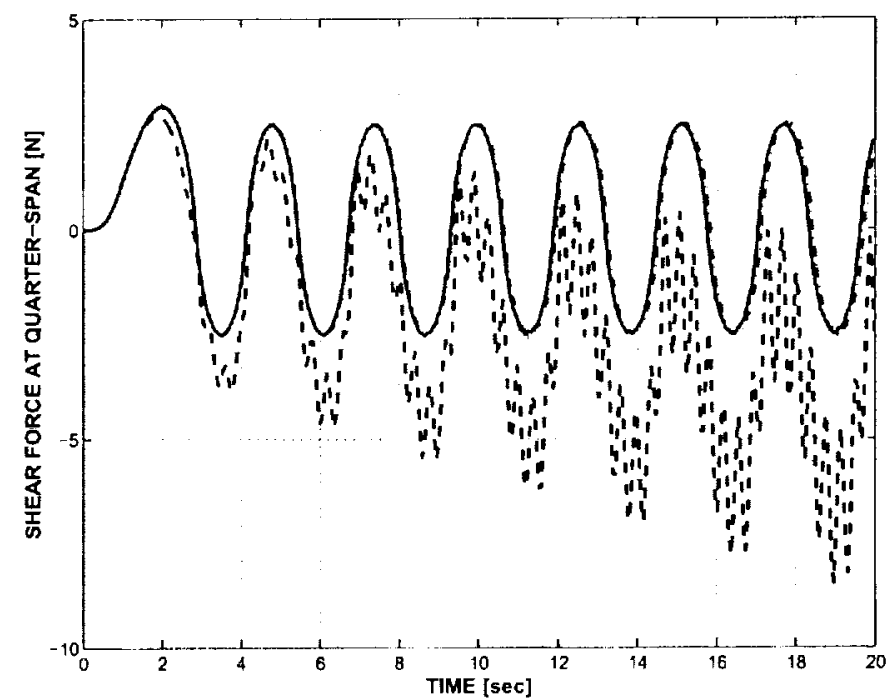

FIG. 5.11. Time history of the quarter-span transverse shear force. Plate model (EDS): solid line; Plate. model (EPS): dashed line; Beam model (FDS): dashed-dot line.

energy predicted in EP simulations. The trajectory of the plate mid-span point is shown in fig. 5.9: good correlation is observed between the predictions of the three models. The beam model is slightly off due to the inherent simplifying assumptions. The behavior of the quarter-span axial force and transverse shear force are shown in fig. 5.10 and 5.11, respectively. The poor predictions of the EP schemes are obvious in these two plots. The history of axial force presents violent oscillations with amplitudes an order of magnitude larger than those observed for the ED scheme. The history of the transverse shear force predicted by the EP scheme quickly diverges from the ED predictions for both beam and plate models. To ascertain the accuracy of the ED predictions, a convergence study was performed. Nearly identical results were found with smaller time step sizes $\Delta t=3.0$ and $1.010^{03} \mathrm{~s}$, or when using the time adaptivity procedure. On the other hand, oscillations of increasing amplitude were found as the time step size is reduced in the EP scheme. Furthermore, the time adaptivity procedure failed to yield any results because the time step size was driven to unreasonably small values, $\Delta t=10^{-07} \mathrm{~s}$, as the procedure tries to cope with increasingly violent oscillations. 


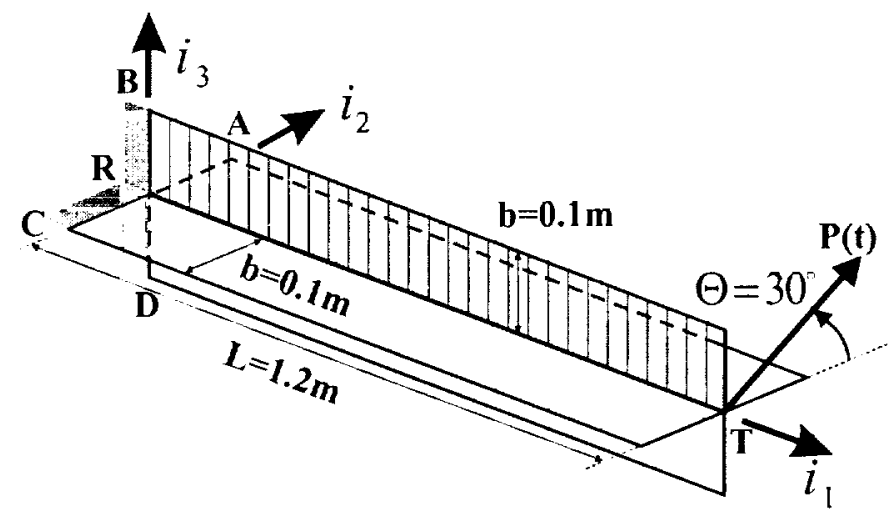

FIG. 5.12. Configuration of the cruciform problem.
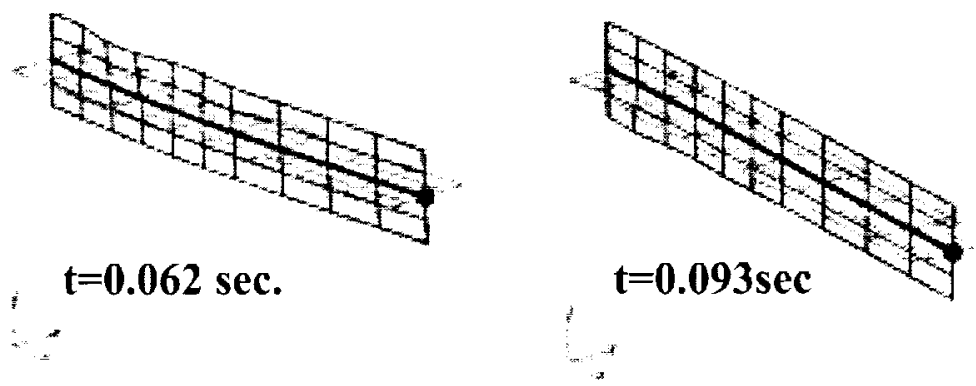

FIG. 5.13. Configuration of the cruciform at times $t=0.062$ and $0.093 \mathrm{~s}$.

5.3. Dynamic Response of a Cruciform. Consider a cruciform consisting of four thin panels (Panels $A, B, C$, and $D$ ) connected to a central beam, as depicted in fig. 5.12. Each panel is of thickness $t=4 \mathrm{~mm}$, length $L=1.2 \mathrm{~m}$, and width $b=0.1 \mathrm{~m}$. The central beam has a square cross-section of width $a=8 \mathrm{~mm}$. A mass $M=12 \mathrm{~kg}$ is attached at the tip of the central beam at point $T$. Panels and beam are simply supported at the root of the cruciform. A concentrated load $P(t)$ is applied at point $T$. The load acts in the plane defined by axes $\underline{i}_{2}$ and $\underline{i}_{3}$ and makes a 30 degree angle with axis $\underline{i}_{2}$. All components are made of aluminum with properties given in the previous example. The time history of the applied load is

$$
P(t)= \begin{cases}P_{0}(1-\cos 2 \pi t / T) / 2 & t \leq T, \\ 0 & t>T,\end{cases}
$$

where $P_{0}=1.2 \mathrm{kN}$ and $T=0.1 \mathrm{~s}$.

As the applied load increases, in-plane stresses in the panels rapidly increase and buckling takes place in those panels subjected to compression, as can be observed in fig. 5.13 that depicts the configuration of the cruciform at two instants in time. The trajectory of point $T$ projected onto plane $\underline{i}_{2}, \underline{i}_{3}$ is shown in fig. 5.14. For reference, the corresponding trajectory of a beam with cross-sectional properties equivalent to those of the cruciform is also presented. Of course, the equivalent beam model is much stiffer since it does not allow buckling to take place. Furthermore, the motion remains confined to the plane defined by axis $\underline{i}_{1}$ and the line of action of the applied load. When each panel is modeled individually, the stiffness of system 


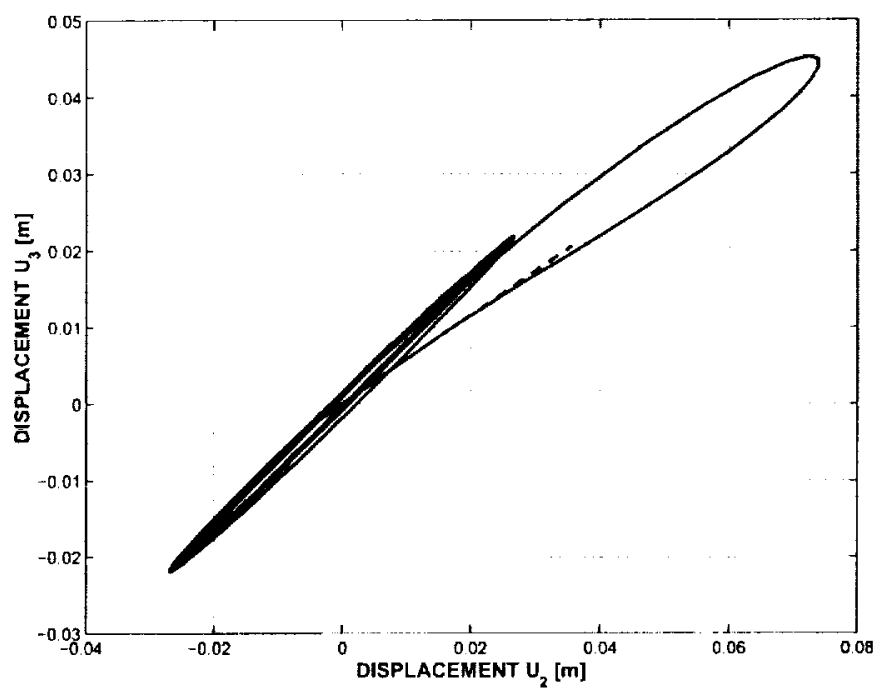

FIG. 5.14. Trajectory of point $T$ of the cruciform projected in the plane $\underline{i}_{2}, \underline{i}_{3}$. Solid line: shell model; dashed line: beam model.

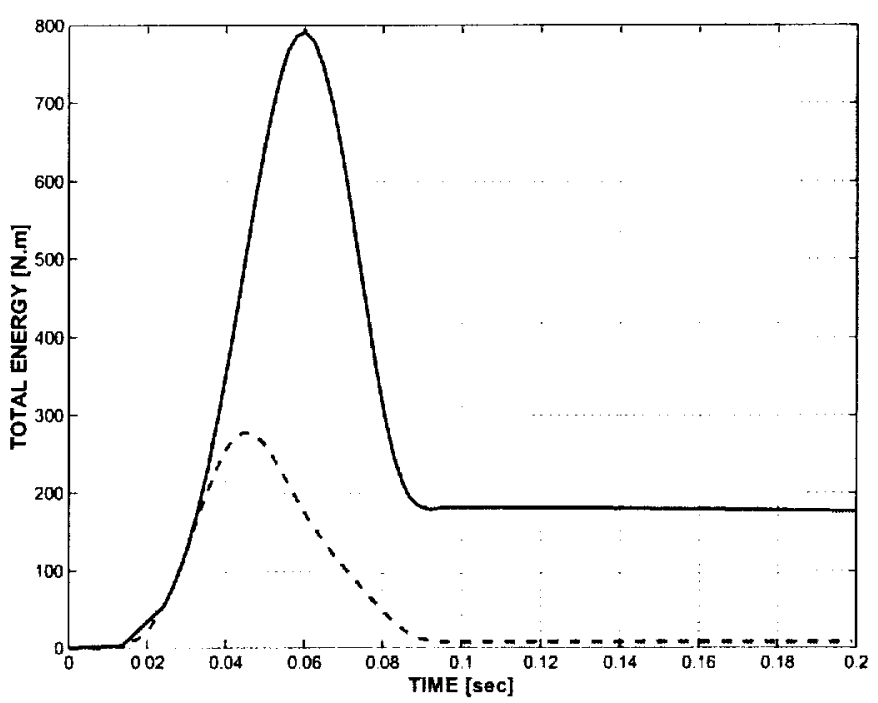

FIG. 5.15. Total mechanical energy of the system. Solid line: shell model; dashed line: beam model.

varies both spatially and temporally, giving rise to the more complex motion shown in fig. 5.14. The total mechanical energy of the system is shown in fig. 5.15. From time $t=0.1$ to $0.2 \mathrm{~s}$, the system is frec and its total mechanical energy should remain constant. Due the dissipative nature of the integration scheme, a small amount of energy is dissipated over that period of time: $2.7 \%$ of the energy was dissipated over the 2435 time step period.

The root shear force and quarter-span bending moment in Panels $A$ and $C$ are shown in fig. 5.16 and 5.17, respectively. Each panel undergoes alternating phases of tensile and compressive loading. During the compressive phases, buckling takes place, and large shear forces and bending moments are observed in contrast with the tensile phases during which these quantities remain much smaller. 


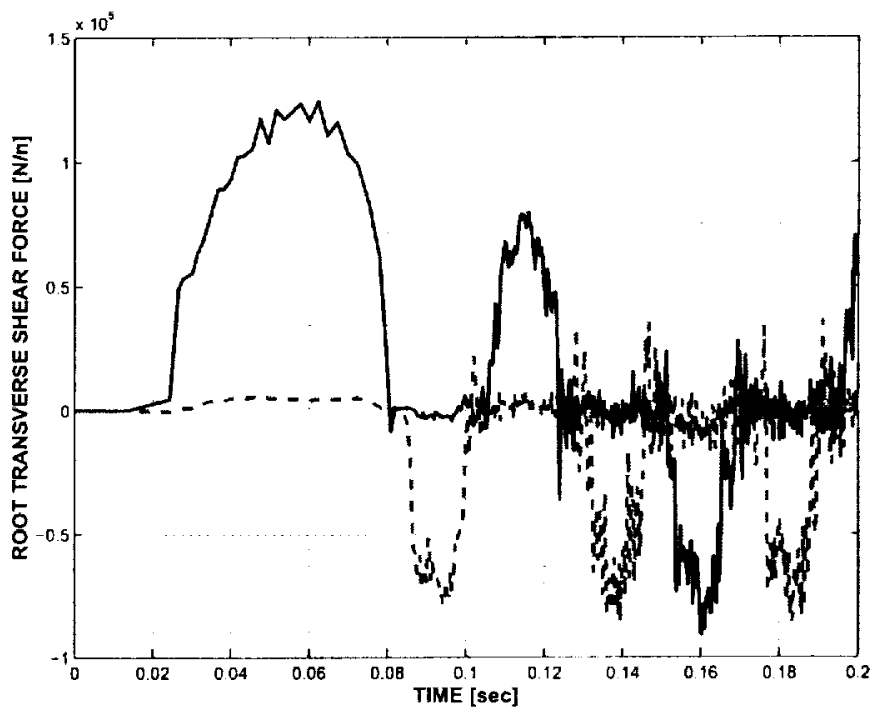

F1G. 5.16. Time history of the root shear force in Panel A (solid line) and Panel $C$ (dashed line).

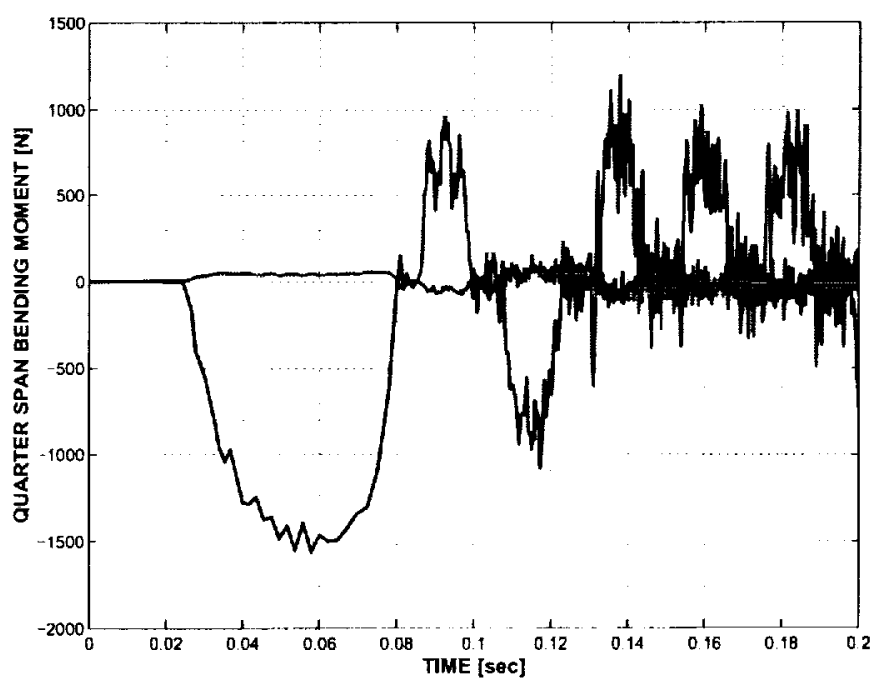

FIG. 5.17. Time history of the quarter-span bending moment in Panel A (solid line) and Panel $C$ (dashed line) 


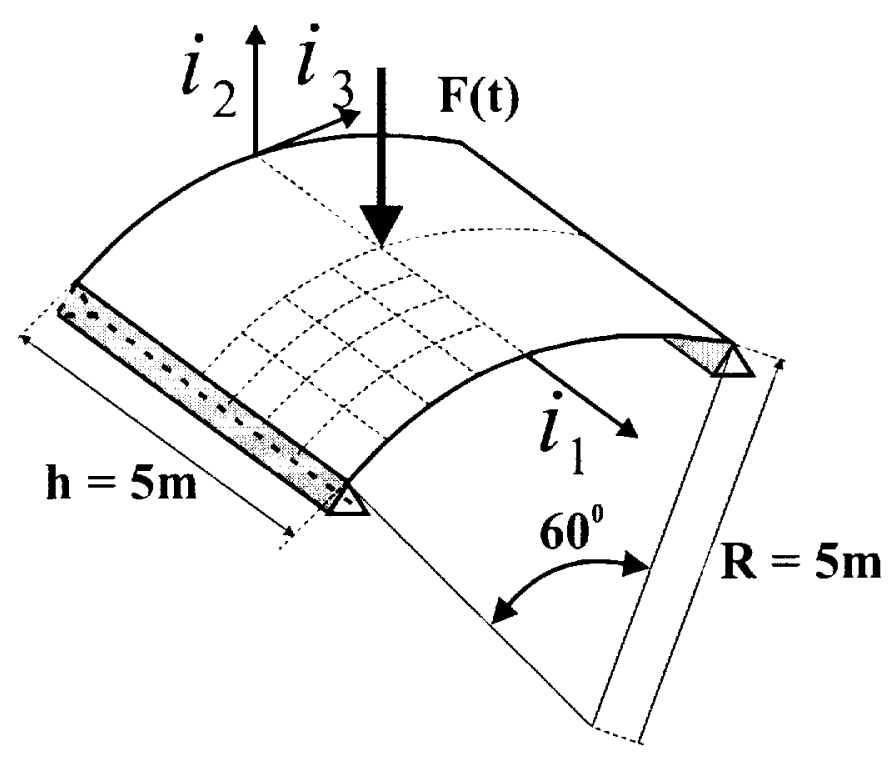

Fic 5.18. Configuration of the snap-through problem.

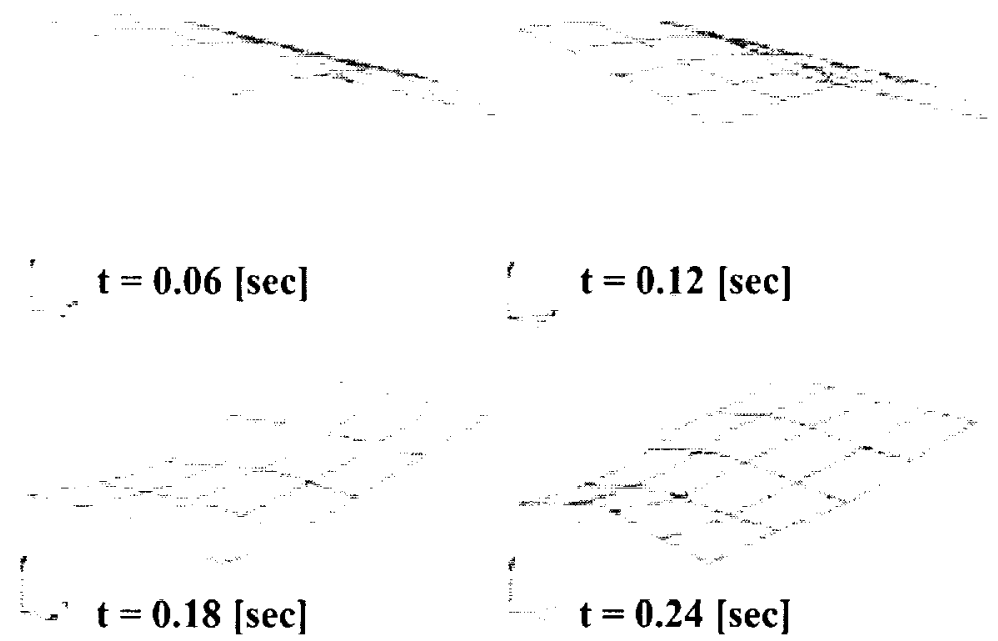

FIG. 5.19. System configurations at various time instants during the simulation.

5.4. Snap-Through of a Cylindrical Shell. The snap-through behavior of a cylindrical shell under a concentrated load was investigated in ref [22]. The shell consists of a 60 degree sector of a cylinder of height $h=5 \mathrm{~m}$, radius $R=5 \mathrm{~m}$ and thickness $t=0.1 \mathrm{~m}$, as shown in fig. 5.18 . Material properties are: Young's modulus $E=210 \mathrm{GPa}$, Poisson ratio $\nu=0.25$ and density $\rho=10^{4} \mathrm{~kg} / \mathrm{m}^{3}$. The two straight edges of the shell are simply supported, while the two curved edges are free.

A concentrated force $F$ is applied at the shell's apex. This force linearly increases from 0 to $510^{7} \mathrm{~N}$ in $0.2 \mathrm{~s}$, then is held constant at that value. The simulation ends at time $t=0.3 \mathrm{~s}$. Due to the symmetry of the problem, a quarter shell only is modeled; a regular $4 \times 4$ mesh of quadratic elements was used. The time step size was selected as $\Delta t=10^{-3} \mathrm{~s}$.

As the load increases, the shell apex displacement increases, then suddenly, snap-through takes place and curvature reverses. Curvature reversal initiates in the region of the applied load, then quickly propagates 


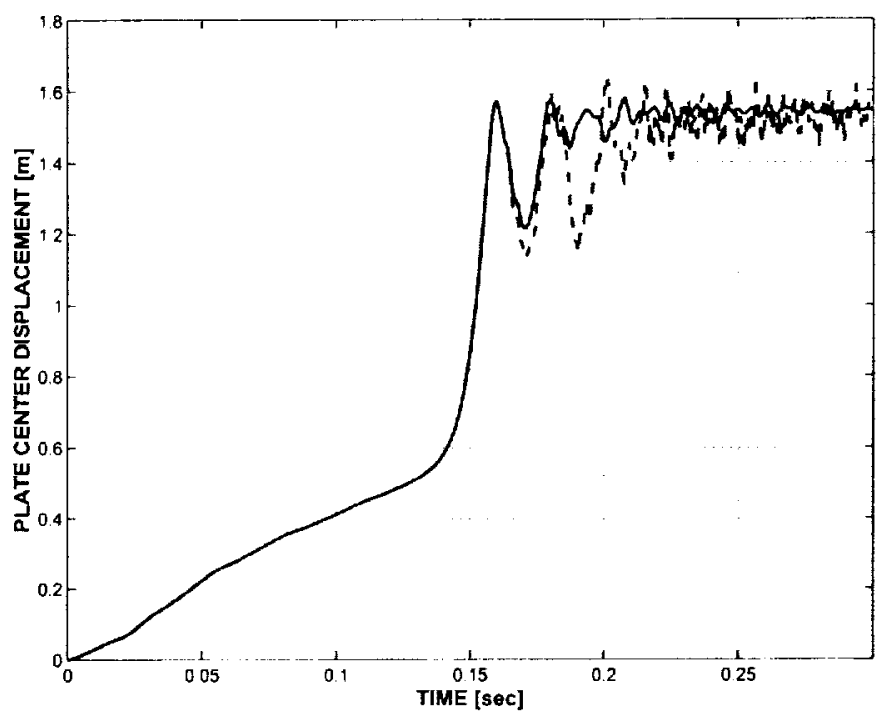

FIG. 5.20. Time history of the plate center vertical displacement. ED scheme: solid line; EP scheme: dashed line.

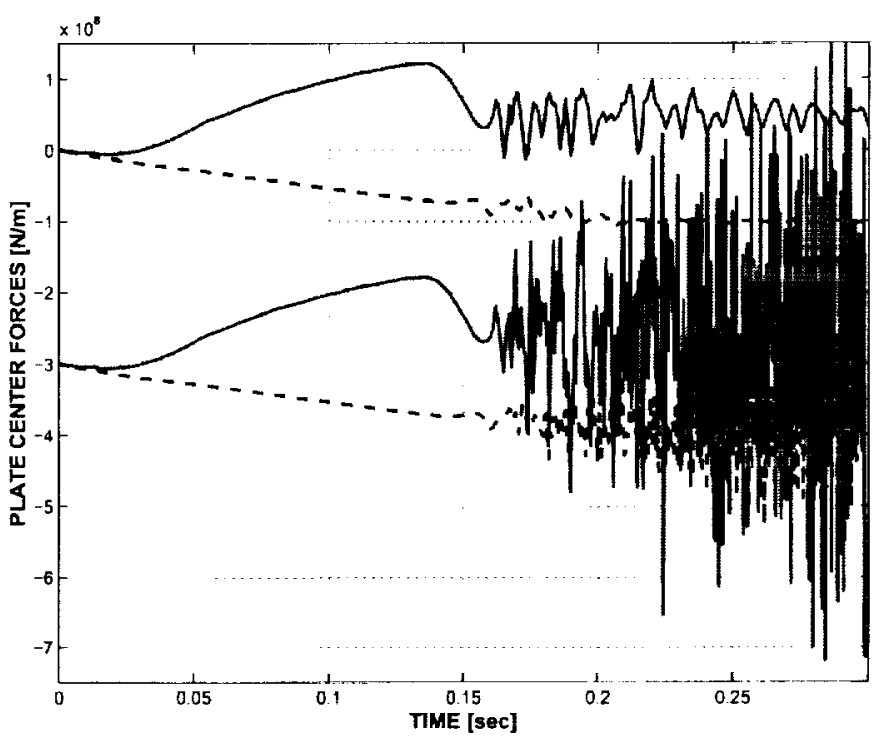

FIG. 5.21. Time history of the plate center forces for the ED and EP schemes. Force $F_{1}:$ solid line; F $F_{3}$ : dashed line. For clarity the EP results are shifted downwards $310^{8} \mathrm{~N}$.

throughout the entire structure, which undergoes subsequent violent oscillations. Snapshots of the system at various instants in time are given in fig. 5.19. The vertical displacements of the point of application of the load computed with the ED scheme is shown in fig. 5.20. Note the gradual increase of the shell deflection, until collapse at buckling and the resulting vibratory response in the inverted configuration.

Ref. [22] presents simulations of this problem using various schemes: the generalized- $\alpha$ [19] and the CEMA [22] schemes. The former scheme features high frequency numerical dissipation and linear stability properties, while the latter adds to the generalized- $\alpha$ method a constraint on the total mechanical energy of the system. CEMA is therefore both energy preserving and high frequency dissipative. The results presented in fig. 5.20 are in close agreement with those obtained with the generalized- $\alpha$ scheme, but quite different 


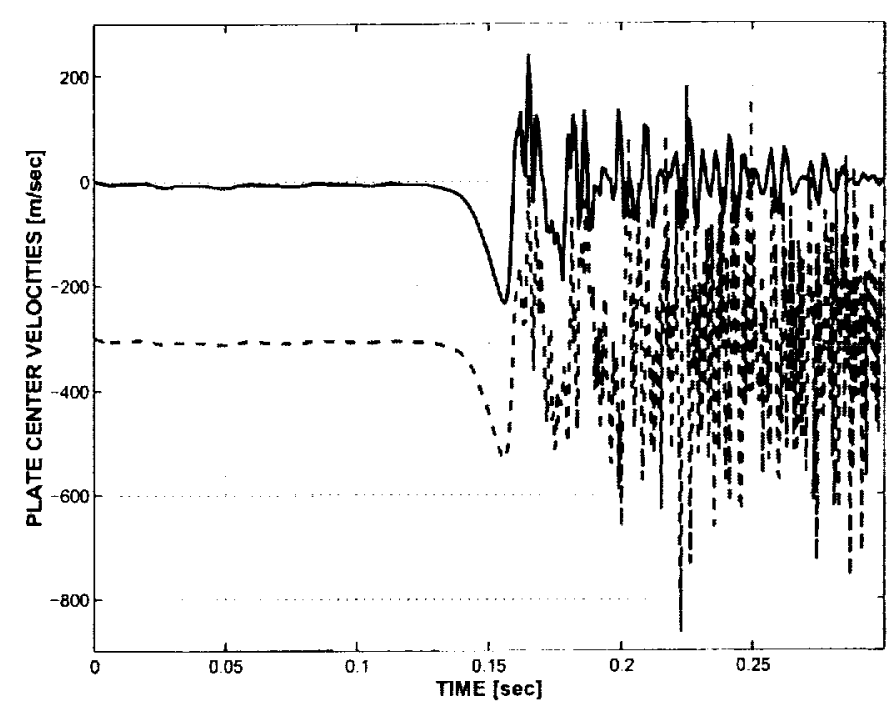

FIG. 5.22. Time history of the plate center vertical velocity. ED scheme: solid line; FP scheme: dashed line, shifted downyards $310^{2} \mathrm{~m} / \mathrm{s}$ for clarity.

from those predicted by CEMA in the post buckling regime. It is important to realize that the higher modes are only an artifact of the discretization process, and should therefore be removed from the computed response. A standard scheme like the generalized- $\alpha$ method accomplishes this goal through the characteristic low-pass shape of its spectral radius; however, there is no guarantee that energy will not be allowed to grow within one step for nonlinear problems. In contrast, CEMA enforces the exact conservation of energy in the nonlinear regime, but at the same time inherits high frequency dissipation from the underlying generalized- $\alpha$ algorithm. Consequently, an artificial mechanism for transferring energy from the higher (artificial) modes to the lower modes is created that drives the response to an erroneous solution. In contrast, the proposed ED scheme achieves both nonlinear stability and high frequency dissipation.

Next, the same problem was simulated with $\rho_{\infty}=1$, i.e. with no high frequency dissipation. In this case, two refinements in time step size were required to successfully complete the simulation, one at time $t=0.1665 \mathrm{~s}\left(\Delta t=510^{-4} \mathrm{~s}\right)$, the other at time $t=0.2142 \mathrm{~s}\left(\Delta t=2.510^{-4} \mathrm{~s}\right)$. Deflections predicted by the FP and ED schemes, shown in fig. 5.20, are in good agreement during the initial snap-through phase, but become increasingly different during the subsequent oscillations. The force and velocity fields are markedly different. The plate center forces for both EP and ED schemes are shown in fig. 5.21. The forces predicted by the EP scheme present violent oscillations of amplitude up to an order of magnitude larger than those predicted by the ED scheme. These violent oscillations hamper the convergence of the Newton process at each time step, leading to the need for smaller time steps. The same observations can be made about fig. 5.22 which compares the plate center vertical velocity. Violent oscillations are initiated at snap-through and the strict preservation of energy implied by the EP scheme prevents any subsequent decay of these vibrations. Since vibratory stresses are a great importance to designers, it is essential to assess the ability of new integration schemes to reliably predict these quantities. It is unfortunate that many scientific publications about geometric integration only present responses for preserved quantities such as total mechanical energy or momentum. The above plots demonstrate that while EP scheme might perform very well for the prediction of total energy, momentum, or even displacement fields, they are unable to reliably predict other important fields such as velocities and internal stresses. Consequently, such schemes 
are of little values in real life applications.

6. Conclusions. In this work, a robust algorithm for the dynamic analysis of geometrically exact shell structures was presented. The method is geometry-based, i.e. it incorporates knowledge about specific qualitative features of the underlying partial differential equations. However, departing from the classical approaches based on strict preservation of energy, the method presented here allows the system to drift away from the level set of constant energy in a controlled and tumable manner.

This feature achieves two goals. First, a bound is placed on the total mechanical energy of the discrete system, leading to the concept of nonlinear unconditional stability; this stability criterion is stronger than that obtained through the classical analysis of numerical schemes. The resulting numerical procedure is endowed with superior robustness, an important feature when dealing with complex engineering problems. Second, the monotonic energy drift is associated with numerical dissipation of the high frequency modes. This tunable dissipation makes the algorithm stiffly accurate, and avoids the build up of energy in the higher modes that are an artifact of the spatial discretization process.

The proposed scheme can deal with general shell structures and is not tied to a specific spatial discretization of the governing partial differential equations. Kinematic nonlinearities are treated in a rigorous manner, and material nonlinearities can be handled when the constitutive laws stem from the existence of a strain energy density function. The efficiency and robustness of the proposed approach were demonstrated with specific numerical examples.

\section{REFERENCES}

[1] K. Bathe and E. Dvorkin, A four-node plate bending element based on mindlin/reissner plate theory and a mixed interpolation, International Journal for Numerical Methods in Engineering, 21 (1985), pp. 367-383.

[2] —- A formulation of general shell elements - the use mixed interpolation of tensorial components, International Journal for Numerical Methods in Engineering, 22 (1986), pp. 697-722.

[3] O. Bauchau, Computational schemes for flexible, nonlinear multi-body systems, Multibody System Dynamics, 2 (1998), pp. 169-225.

[4] - On the modeling of friction and rolling in flexible multi-body systems, Multibody System Dynamics, 3 (1999), pp. 209-239.

[5] - Analysis of flexible multi-body systems with intermittent contacts, Multibody System Dynamics, 4 (2000), pp. 23-54.

[6] O. BauchaU AND C. BotTasso, On the design of energy preserving and decaying schemes for flexible, nonlinear multi-body systems, Computer Methods in Applied Mechanics and Engineering, 169 (1999), pp. 61-79.

[7] — Robust integration schemes for flexible multibody systems, Computer Methods in Applied Mechanics and Engineering, (2000).

[8] O. Bauchat, J. ChOI, and C. Bottasso, On the modeling of shells in multibody dynamics, Multibody System Dynamics, (2000).

[9] O. BAUCHAU AND T. JOO, Computational schemes for nonlinear elasto-dynamics, International Journal for Numerical Methods in Fngineering, 45 (1999), pp. 693-719.

[10] O. Bavchau and N. Throron, Energy deraying scheme for non-linear beam models, Computer Methods in Applied Mechanics and Engineering, 134 (1996), pp. 37-56. 
[11] _ Energy decaying schemes for nonlinear elastic multi-body systems, Computers and Strurtures, 59 (1996), pp. 317331.

[12] M. Borri, C. BotTtasso, AND L. Thainfid, A novel momentum-preserving energy-decaying algorithm for finite element multibody procedures, in Proceedings of Computational Aspects of Nonlinear Structural Systems with Large Rigid Body Motion, NATO Advanced Research Workshop. Pultusk, Poland, July 2-7, 2000 .

[13] M. Borri, L. TRanelu, AND C. BotTasso, On representations and parameterizations of motion, Multibody Systems Dynamics, 4 (2000), pp. 129193.

[14] C. BOTTASSO AND M. BORRI, Energy preserving/decaying schemes for non-linear beam dynamics using the helicoidal approximation, Computer Methods in Applied Mechanics and Engineering, 143 (1997), pp. 393415 .

[15] — Integrating finite rotations, Computer Methods in Applied Mechanics and Engincering, 164 (1998), pp. 307331.

[16] C. Bottasso, M. Borrt, and L. Trainfi,di, Integration of elastic multibody systems using invariant preserving/dissipating schemes. Part I formulation \& Part. II numerical schemes and applications., Computer Methods in Applied Mechanics and Engineering, (2000).

[17] M. BUCALFm AND K. BATHF, Higher-order mitc general shell elements, International Journal for Numerical Methods in Engineering, 36 (1993), pp. 3729 3754.

[18] C. BUDD AND A. ISFRI,Fs, Geometric integration: Numerical solution of differential equations on manifolds, Philosophical Transactions of the Royal Society of London Series A-Mathematical Physical and Engineering Sciences, 357 (1999), pp. 945-956.

[19] J. Chung and G. HulbrRt, A time integration algorithm for structural dynamics with improved numerical dissipation: The generalized- $\alpha$ method, Journal of Applied Mechanics, 122 (1995), pp. 254 266.

[20] H. HILBF, T. HughFs, AND R. TAYLOR, Improved numerical dissipation for time integration algorithms in structural dynamics, Earthquake Engineering and Structural Dynamics, 5 (1977), pp. 282292.

[21] T. Kane and D. Levinson, Dynamics: Theory and Applications, McGraw-Hill, Inc., New York, 1985.

[22] D. KUHL AND R. E., Constraint energy momentum algorithm and its application to non-linear dynamics of shells, Computer Methods in Applied Mechanics and Engineering, 136 (1996), pp. 293315.

[23] J. SIMO AND N. TARNOW, The discrete energy-momentum method. conserving algorithms for nonlinear dynamics, ZAMP, 43 (1992), pp. 757-792.

[24] _ A new energy and momentum conserving algorithm for the nonlinear dynamics of shells, International Journal for Numerical Methods in Engineering, 37 (1994), pp. 2527-2549.

[25] J. Simo, N. TaRnow, AND M. DobI.ARF, Non-linear dynamics of three-dimensional rods: Exact energy and momentum conserving algorithms, International Journal of Numerical Methods in Engineering, 38 (1995), pp. 14311473

[26] J. SimO AND K. WONG, Unconditionally stable algorithms for rigid body dynamics that exactly preserve energy and momentum, International Journal for Numerical Methods in Engineering, 31 (1991), pp. $19-52$. 


\section{Appendix A. Rodrigues Parameters.}

A common representation of finite rotations [21] is in terms of Rodrigues parameters $\underline{r}=2 \underline{k} \tan \phi / 2$, where $\phi$ is the magnitude of the finite rotation and $\underline{k}$ the components of the unit vector about which it takes place. The following notation is introduced $r_{0}=\cos ^{2} \phi / 2=1 /\left(1+\underline{r}^{T} \underline{r} / 4\right)$, and the finite rotation tensor $R$ then writes

$$
R(\underline{r})=I+r_{0} \widetilde{r}+\frac{r_{0}}{2} \widetilde{r} \widetilde{r}
$$

The following decomposition of the rotation tensor is extensively used in this work

$$
R=\left(I+\frac{\bar{r}}{2}\right)\left(I+\frac{\tilde{r}}{2}\right)^{-T}=\left(I+\frac{\tilde{r}}{2}\right)^{-T}\left(I+\frac{\tilde{r}}{2}\right) ; \quad\left(I+\frac{\tilde{r}}{2}\right)^{-T}=\frac{R+I}{2}
$$

\section{Appendix B. Orientation of a Unit Director.}

Consider a unit vector $\underline{i}_{3}$, called a director, that rotates to a final orientation $\underline{e}_{3}$. For convenience, this director is considered to be the third unit vector of a triad $\mathcal{S}$ defined by $\underline{i}_{1}, \underline{i}_{2}, \underline{i}_{3}$, rotating to a triad $\mathcal{S}^{*}$ with orientation $\underline{e}_{1}, \underline{e}_{2}, \underline{e}_{3}$. The relationship between these two triads is $\underline{e}_{\alpha}=R \underline{i}_{\alpha}$, where $R$ is an orthogonal rotation tensor. If one solely focuses on the director, this rotation tensor is not uniquely defined, as any rotation about the director leaves its orientation unchanged. A virtual change in the director orientation is

$$
\delta \underline{e}_{3}=\widetilde{e}_{3}^{T} \underline{\delta \psi}
$$

where $\delta \psi$ is the virtual rotation vector, $\widetilde{\delta \psi}=\delta R R^{T}$.

The components of the virtual change in director orientation measured in $S^{*}$ become

$$
R^{T} \delta \underline{e}_{3}=R^{T} \tilde{e}_{3}^{T} \underline{\delta \psi}=\widetilde{i}_{3}^{T} R^{T} \underline{\delta \psi}=\widetilde{i}_{3}^{T} \underline{\delta \psi^{*}}=\left|\begin{array}{c}
-\delta \psi_{2}^{*} \\
\delta \psi_{1}^{*} \\
0
\end{array}\right|,
$$

where $\delta \psi^{*}$ are the components of the virtual rotation vector in $\mathcal{S}^{*}$. This relationship clearly demonstrates that arbitrary values of $\delta \psi_{3}^{*}$, corresponding to virtual rotations of the director about its own orientation, will not affect virtual changes in the director orientation, and hence, setting $\delta \psi_{3}^{*}=0$ is a valid choice. The following notation is adopted

$$
\underline{\delta \psi^{*}}=\underline{i}_{1} \delta \alpha_{1}^{*}+\underline{i}_{2} \delta \alpha_{2}^{*}=b \underline{\delta \alpha^{*}} ; \quad b=\left[\underline{i}_{1}, \underline{i}_{2}\right] .
$$

$\underline{\delta \alpha}^{*}$ is a $2 \times 1$, "two parameter" virtual rotation vector. It follows that $\underline{\delta \psi}=R \underline{\delta \psi^{*}}=R b \underline{\delta \alpha^{*}}$, and hence

$$
\delta \underline{e}_{\alpha}=R \widetilde{i}_{\alpha}^{T} b \underline{\delta \alpha}^{*} .
$$

If Rodrigues parameters are used to parameterize $R$, an equivalent expression can be obtained for finite changes in director orientation with the help of eq. (A.2)

$$
\underline{e}_{\alpha f}-\underline{e}_{\alpha i}=R_{m} \widetilde{i}_{\alpha}^{T} b \underline{s}^{*}=Q_{m} \underline{s}^{*} ; \quad \underline{r}^{*}=b \underline{s}^{*},
$$

where $\underline{r}^{*}$ are the Rodrigues parameter measured in $\mathcal{S}^{*}$, and $\underline{s}^{*}$ the corresponding "two parameter" incremental rotation vector. 


\begin{tabular}{|c|c|c|c|}
\hline \multicolumn{3}{|c|}{ REPORT DOCUMENTATION PAGE } & $\begin{array}{l}\text { Form Approved } \\
\text { OMB No. 0704-0188 }\end{array}$ \\
\hline \multicolumn{4}{|c|}{ 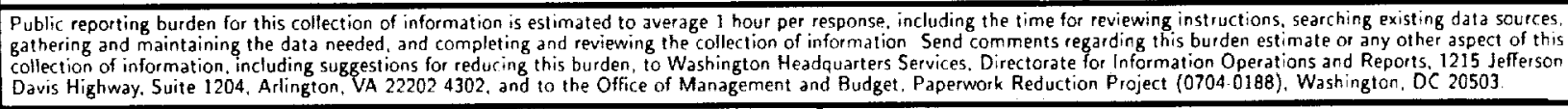 } \\
\hline 1. AGENCY USE ONLY(Leave blank) & $\begin{array}{l}\text { 2. REPORT DATE } \\
\text { November } 2000\end{array}$ & \multicolumn{2}{|c|}{$\begin{array}{l}\text { 3. REPORT TYPE AND DATES COVERED } \\
\text { Contractor Report }\end{array}$} \\
\hline \multicolumn{3}{|c|}{$\begin{array}{l}\text { 4. TITLE AND SUBTITLE } \\
\text { An energy decaying scheme for nonlinear dynamics of shells }\end{array}$} & \multirow[t]{2}{*}{$\begin{array}{l}\text { 5. FUNDING NUMBERS } \\
\text { C NAS1-97046 } \\
\text { WU } 505-90-52-01\end{array}$} \\
\hline \multicolumn{3}{|c|}{$\begin{array}{l}\text { 6. AUTHOR(S) } \\
\text { Carlo L. Bottasso, Olivier A. Bauchan, and Jon-Young Choi }\end{array}$} & \\
\hline \multicolumn{2}{|c|}{$\begin{array}{l}\text { 7. PERFORMING ORGANIZATION NAME(S) AND ADDRESS(ES) } \\
\text { ICASE } \\
\text { Mail Stop 132C } \\
\text { NASA Langley Research Center } \\
\text { Hampton, VA } 23681-2199\end{array}$} & & $\begin{array}{l}\text { 8. PERFORMING ORGANIZATION } \\
\text { REPORT NUMBER } \\
\text { ICASE Report No. } 2000-43\end{array}$ \\
\hline \multicolumn{3}{|c|}{$\begin{array}{l}\text { 9. SPONSORING/MONITORING AGENCY NAME(S) AND ADDRESS(ES) } \\
\text { National Aeronautics and Space Administration } \\
\text { Langley Research Center } \\
\text { Hampton, VA } 23681-2199\end{array}$} & $\begin{array}{l}\text { 10. SPONSORING/MONITORING } \\
\text { AGENCY REPORT NUMBER } \\
\text { NASA/CR-2000-210557 } \\
\text { ICASE Report No. } 2000-43\end{array}$ \\
\hline \multicolumn{4}{|c|}{$\begin{array}{l}\text { 11. SUPPLEMENTARY NOTES } \\
\text { Langley Technical Monitor: Dennis M. Bushnell } \\
\text { Final Report } \\
\text { Submitted to Computer Methods in Applied Mechanics and Engineering. }\end{array}$} \\
\hline \multicolumn{2}{|c|}{$\begin{array}{l}\text { 12a. DISTRIBUTION/AVAILABILITY STATEMENT } \\
\text { Unclassified-Unlimited } \\
\text { Subject Category } 64 \\
\text { Distribution: Nonstandard } \\
\text { Availability: NASA-CASI (301) 621-0390 }\end{array}$} & 12b. DISTI & 12b. DISTRIBUTION CODE \\
\hline \multicolumn{4}{|c|}{$\begin{array}{l}\text { 13. ABSTRACT (Maximum } 200 \text { words) } \\
\text { A novel integration scheme for nonlinear dynamics of geometrically exact shells is developed based on the inextensible } \\
\text { director assumption. The new algorithm is designed so as to imply the strict decay of the system total mechanical } \\
\text { energy at each time step, and consequently unconditional stability is achieved in the nonlinear regime. Furthermore, } \\
\text { the scheme features tunable high frequency numerical damping and it is therefore stiffy accurate. The method is } \\
\text { tested for a finite element spatial formulation of sliells based on mixed interpolations of strain tensorial components } \\
\text { and on a two-parameter representation of director rotations. The robustness of the scheme is illustrated with the } \\
\text { help of numerical examples. }\end{array}$} \\
\hline \multirow{2}{*}{\multicolumn{3}{|c|}{$\begin{array}{l}\text { 14. SUBJECT TERMS } \\
\text { geometrically exact shell, time integration, energy decaying scheme }\end{array}$}} & \begin{tabular}{|c|} 
15. NUMBER OF PAGES \\
28 \\
\end{tabular} \\
\hline & & & $\begin{array}{r}\text { 16. PRICE CODE } \\
\mathrm{A} 03 \\
\end{array}$ \\
\hline $\begin{array}{l}\text { 17. SECURITY CLASSIFICATION } \\
\text { OF REPORT } \\
\text { Unclassified }\end{array}$ & $\begin{array}{l}\text { 18. SECURITY CLASSIFICATION } \\
\text { OF THIS PAGE } \\
\text { Unclassified }\end{array}$ & $\begin{array}{l}\text { 19. SECURITY CLASSIFICATION } \\
\text { OF ABSTRACT }\end{array}$ & $\begin{array}{l}\text { 20. LIMITATION } \\
\text { OF ABSTRACT }\end{array}$ \\
\hline
\end{tabular}

